\title{
Primeros conodontos del Frasniense en el Complejo Maláguide de Granada (Cordillera Bética)
}

\author{
Ángela CABRERA-PORRAS ${ }^{*}$, Rosario RODRÍGUEZ-CANERO \& Agustín MARTÍN- \\ ALGARRA ${ }^{3}$
}

${ }^{1}$ C/ Lope de Vega, 6. 14300 Villaviciosa de Córdoba; angelacabrera5@correo.ugr.es

${ }^{2}$ Avda. de Andalucía, 25, 11A. 29006 Málaga; charorc@ugr.es

${ }^{3}$ Avda. Severo Ochoa s/n. Dpto. Estratigrafía y Paleontología. Universidad de Granada 18071 Granada; agustin@ugr.es

*Corresponding author

Cabrera-Porras, Á., Rodríguez-Cañero, R. \& Martín-Algarra, A. 2019. Primeros conodontos del Frasniense en el Complejo Maláguide de Granada (Cordillera Bética). [First Frasnian conodonts in the Malaguide Complex of Granada (Betic Cordillera)]. Spanish Journal of Palaeontology, 34 (2), 163-186.

Manuscript received 26 November 2018

Manuscript accepted 13 May 2019 https://doi 10.7203/sjp.34.2.16092

(C) Sociedad Española de Paleontología ISSN 2255-0550

\section{Resumen}

Un delgado nivel calcáreo, localizado en la unidad tectónica más alta del Complejo Maláguide en la provincia de Granada, ha proporcionado una abundante fauna de conodontos (808 elementos por $\mathrm{kg}$ de muestra) integrada por Ancyrodella binodosa, Ancyrodella pristina, Icriodus difficilis, Icriodus symmetricus, Polygnathus decorosus, Polygnathus dengleri y Polygnathus dubius, entre otras especies. Esta asociación corresponde a una biofacies de polygnáthidos y ha permitido asignar este nivel a las zonas MN1 y MN2 (Frasniense inicial), cuya presencia ha sido constatada por vez primera en el Complejo Maláguide. Esta es, asimismo, la primera datación del Frasniense en el sector central de la Cordillera Bética. El nivel se intercala entre pelitas con niveles areniscosos, volcanoclásticos y otros delgados horizontes carbonatados del Emsiense y del Fameniense, que yacen bajo la Fm. Falcoña (Carbonífero inferior). Esta sucesión pre-Falcoña se encuentra en una unidad tectónica diferente a la que incluye la Fm. Santi Petri, con la que se correlaciona, aunque se depositó en ambientes menos distales y menos profundos que dicha formación, que es la más extensamente representada y característica del Devónico Maláguide.

Palabras clave: conodontos, biofacies de polygnáthidos, zonas MN1 y MN2, sucesión pre-Falcoña.

\begin{abstract}
A thin calcareous bed found in the highest tectonic unit of the Malaguide Complex near Granada has provided a very rich conodont fauna (808 elements per $\mathrm{kg}$ of sample) formed by Ancyrodella binodosa, Ancyrodella pristina, Icriodus difficilis, Icriodus symmetricus, Polygnathus decorosus, Polygnathus dengleri, and Polygnathus dubius, among other species. This association corresponds to a polygnathid biofacies and it allows its accurate dating as belonging to the MN1 and MN2 zones (earliest Frasnian), whose presence has been found for the first time in the Malaguide Complex. Furthermore, this is the first biostratigraphic age of Frasnian beds in the central sector of the Betic Cordillera. The horizon is interbedded between pelites including sandstone, volcaniclastic and other thin calcareous beds, dated as Emsian and Famennian, which underlie the Falcoña Fm. (lower Carboniferous). This pre-Falcoña succession is located within a different tectonic unit to that bearing the Santi Petri Fm. Both successions are equivalent, but the former was deposited in shallower and more proximal sedimentary environments than the latter, which is the most typical and widest outcropping Devonian Malaguide succession.
\end{abstract}

Keywords: conodonts, polygnathid biofacies, MN1 y MN2 zones, pre-Falcoña succession. 


\section{INTRODUCCIÓN}

Diversos trabajos reconocen la existencia del Frasniense, que ha sido identificado también mediante fauna de conodontos, en distintas localidades de la Península Ibérica: i) en los Pirineos (Boersma, 1973; Liao et al., 2001; Sanz-López, 2002; Sánchez de Posada et al., 2007; Liao \& Valenzuela-Ríos, 2012); ii) en la Cordillera Ibérica (Carls \& Valenzuela-Ríos, 2002); y iii) en la Cordillera Cantábrica (Adrichem Boogaert, 1967; García-López, 1976; Raven, 1983; García-López, 1987; Sanz-López et al., 1999; García-López \& Sanz-López, 2002). De igual forma, Kockel \& Stoppel (1962) dataron, por primera vez en la Cordillera Bética, un nivel del Frasniense en el Complejo Maláguide (Fig. 1a) de la región de Almogía (Málaga). En afloramientos maláguides del área de Vélez Rubio, Van de Boogaard (1965) identificó un horizonte del Frasniense superior-Fameniense inferior. Otros niveles del Frasniense han sido datados posteriormente, también mediante conodontos, en afloramientos del Complejo Maláguide en la provincia de Málaga: i) de nuevo en la zona de Almogía (Rodríguez-Cañero, 1993; Rodríguez-Cañero et al., 2010); ii) en clastos incluidos en el conglomerado de Retamares del Carbonífero inferior en Fuengirola (Herbig, 1984; Rodríguez-Cañero, 1993); iii) en el arroyo de la Cruz (Marbella), donde Herbig (1985) reconoció la existencia de un Frasniense superior; y iv) en clastos incluidos en un conglomerado del Devónico Superior, relacionado con los eventos del límite Frasniense-Fameniense, en el Cortijo de la Falcoña (Rodríguez-Cañero \& Martín-Algarra, 2014).

El objetivo de este trabajo es dar a conocer la primera datación precisa realizada hasta la fecha de un horizonte del Frasniense en el Complejo Maláguide de la provincia de Granada (Fig. 1b). Dicho horizonte es, además, el nivel más antiguo del Devónico Superior reconocido hasta la fecha en todo el Complejo Maláguide. El hallazgo permite correlacionar, con mayor precisión, el área estudiada con otras sucesiones mejor conocidas en otras zonas de este Complejo.

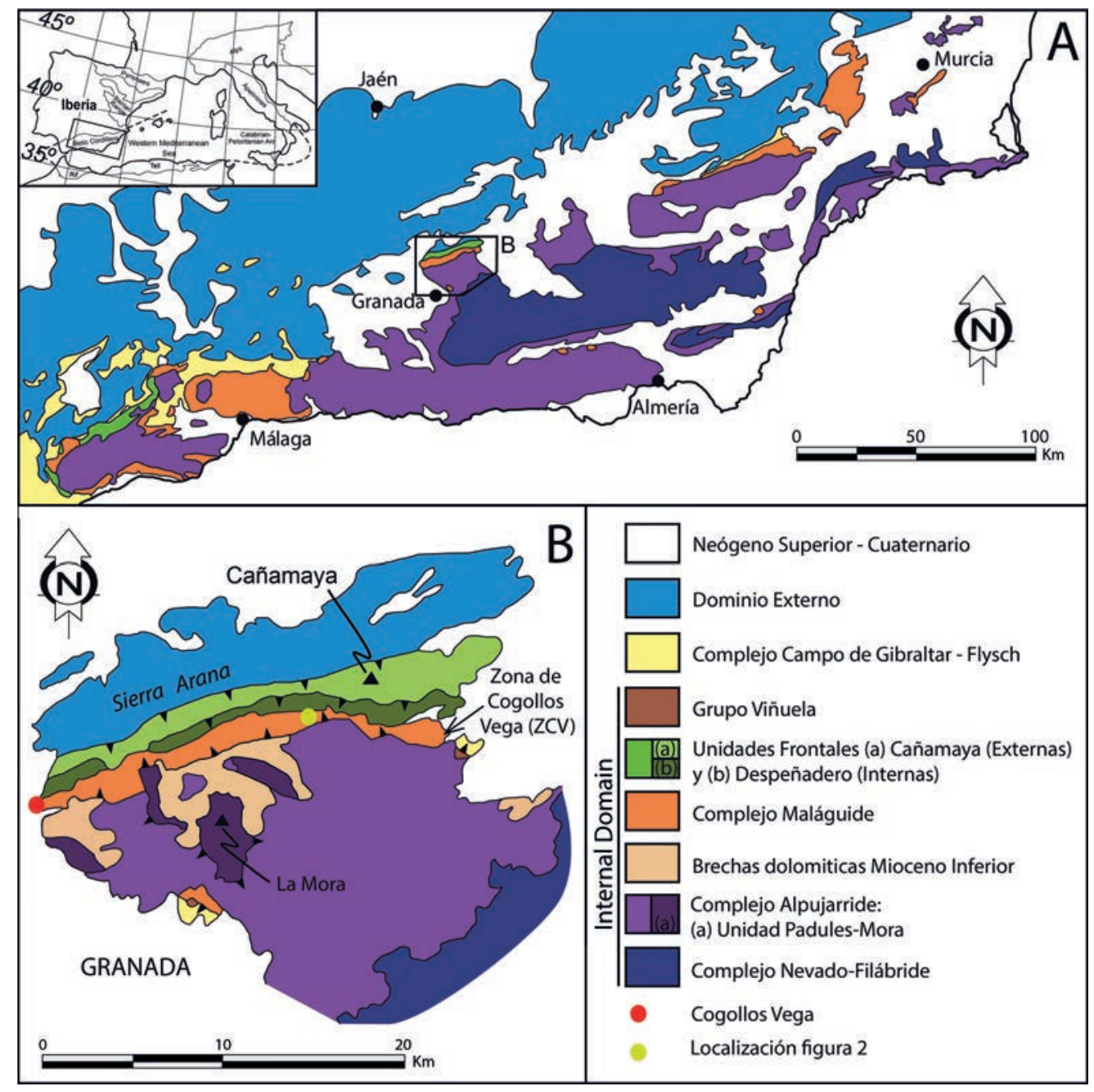

Figura 1. El Complejo Maláguide en el sector central de la Cordillera Bética (provincia de Granada). a) Mapa geológico general de la Cordillera Bética con sus principales dominios. b) Unidades tectónicas principales de la zona de estudio (modificada de Navas-Parejo, 2012). 


\section{MARCO GEOLÓGICO}

\subsection{La Zona de Cogollos Vega}

El mayor afloramiento del Complejo Maláguide (Blumenthal, 1927; Durand-Delga, 1968) en la provincia de Granada aparece en las inmediaciones del contacto entre las Zonas Internas y Externas de la Cordillera Bética (Fig. 1a), formando una banda estrecha de aproximadamente 20 kilometros de largo y de 1-2 kilometros de anchura que Blumenthal \& Fallot (1935) denominaron Zona de Cogollos Vega (ZCV: Fig. 1b). En esta provincia existen también otros afloramientos maláguides de menor entidad situados unos 10 kilometros al sur de la ZCV (García-Dueñas \& Navarro-Vilá, 1976; Navarro-Vilá \& García-Dueñas, 1979), en las inmediaciones de la localidad de Beas de Granada y entre Diezma y La Peza (Fig. 1b), así como otros al sur de Sierra Nevada, en el denominado Corredor de las Alpujarras (Fig. 1a). Todos ellos constituyen un testimonio del amplio recubrimiento que presentaba este complejo sobre los subyacentes, antes de su casi completa erosión.

Los materiales paleozoicos de la ZCV pertenecen al Grupo Piar (Martín-Algarra, 1987; Martín-Algarra et al., 2004). Este grupo aparece representado en la zona en su totalidad con sus formaciones tradicionales, con la excepción (con algunas reservas) de la Fm. Morales. Los rasgos principales de las formaciones clásicas del Grupo Piar encontradas en la zona se presentan a continuación.

\subsubsection{Formación Santi Petri (Michelau, 1942,} Mon, 1971)

Se reconoce principalmente por la presencia de las facies denominas calizas alabeadas (Orueta, 1917). Son calizas finamente detríticas asociadas a un sistema turbidítico distal, que están habitualmente muy replegadas. Sus facies van de puramente terrígenas de grano fino a grauvacas y pelitas ligeramente calcáreas o a calizas finamente detríticas, en general de tonalidades verde-oliváceas, más amarillentas por meteorización. En sí misma, la Fm. Santi Petri carece de fósiles, pero se le ha atribuido una edad pre-Carbonífera por encontrarse estratigráficamente debajo de niveles bien datados de la Fm. Falcoña, del Carbonífero inferior.

\subsubsection{Formación Falcoña (Herbig, 1983)}

En el área estudiada no supera los 10 metros de potencia, pero presenta dos miembros bien diferenciados: i) unas radiolaritas negras (liditas), que a menudo aparecen decoloradas y con tonos anaranjados o amarillentos y que han sido datadas con radiolarios como Tournasiense en los Montes de Málaga (O'Dogherty et al., 2000); y ii) unas calizas azuladas de edad Viseense superior que reposan sobre las anteriores (Rodríguez-Cañero \& GuerraMerchán, 1996; Navas-Parejo et al., 2015). Estos materiales constituyen el mejor nivel guía del Paleozoico Maláguide.

\subsubsection{Formación Almogía (Mon, 1971)}

Se compone de sedimentos turbidíticos sinorogénicos, depositados durante el inicio de la Orogenia Varisca (facies Culm). Se trata de una megasecuencia granodecreciente, que comienza con grauvacas en bancos gruesos con ocasionales niveles de conglomerados (Miembro Retamares: Kockel \& Stoppel, 1962) sobre los que se depositó una alternancia de pizarras oliváceas con algunos niveles de areniscas. Es la formación mejor desarrollada en la ZCV superando en muchas zonas los 100 metros de espesor.

\subsubsection{Formación Marbella (Michelau, 1942, Blumenthal, 1949; Herbig, 1984)}

Se encuentra disconforme sobre la unidad anterior y se compone principalmente de conglomerados depositados por flujos de derrubios de matriz arenosa, con clastos muy heterométricos y de diferentes litologías: filitas, cuarcitas, pizarras y liditas, además de calizas con fósiles de aguas someras, granitos y gneises, siendo los tres últimos litotipos de procedencia desconocida. Su potencia en la ZCV supera localmente los 30 metros.

\subsection{Unidades tectónicas presentes en la ZCV}

Desde un punto de vista estructural, la ZCV se organiza en tres grandes unidades tectónicas, con rasgos equivalentes a los de las unidades maláguides reconocidas por autores previos en el área de Vélez Rubio (Roep \& Mac Gillavry, 1960) y Ardales (Martín-Algarra et al., 2009a, 2009b). Martín-Algarra (1987) y Navas-Parejo (2012) las denominaron, de abajo a arriba tectónicamente, Unidad Maláguide Epimetamórfica, Unidad Maláguide Inferior y Unidad Maláguide Superior (Fig. 2). Aunque las sucesiones estratigráficas de las tres unidades muestran grandes semejanzas a rasgos generales, en el detalle sus características litoestratigráficas varían, tanto en lo que se refiere a los terrenos del Paleozoico como a los más modernos, por lo que sus sucesiones se describirán independientemente a continuación.

\subsubsection{Unidad Maláguide Epimetamórfica (UME)}

Es equivalente a la más alta de las denominadas "unidades de transición" alpujárride-maláguide de Sanz-Galdeano et al. (1995). Se sitúa directamente sobre el Complejo Alpujárride y es la única que está afectada por un metamorfismo alpino perceptible, aunque de bajo grado. 


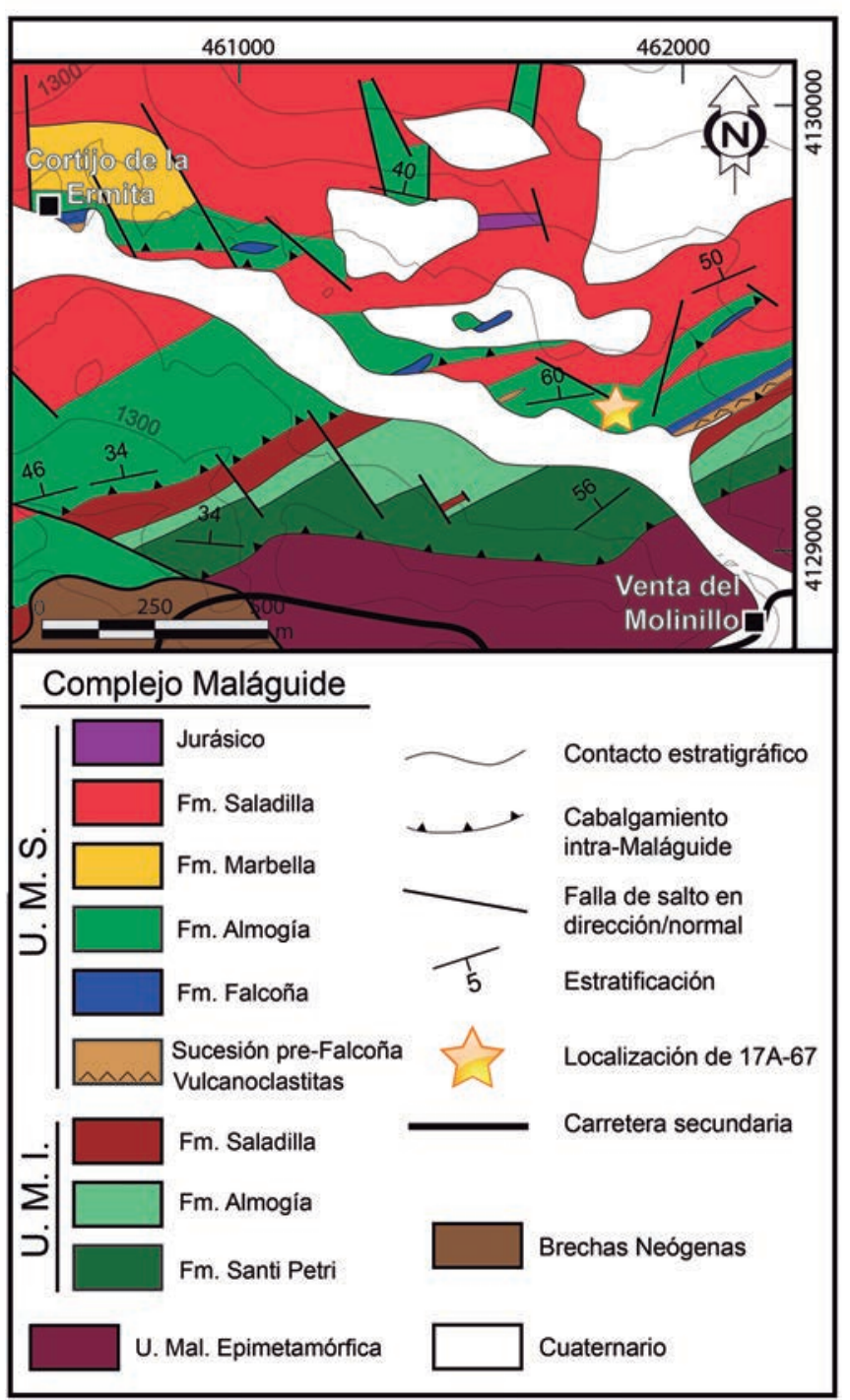

Figura 2. Mapa geológico del área de estudio (coordenadas UTM). La estrella señala la posición de la sección del Frasniense estudiada en este trabajo.

Por ello, aún se reconocen características originales de su sucesión estratigráfica. Esta se inicia con metapelitas y metapsammitas pardas de rasgos afines a la Fm. Almogía. Sobre ellas aparecen materiales detríticos rojos continentales del Triásico de la Fm. Saladilla (Roep, 1972), que el metamorfismo ha transformado en filitas y cuarcitas rojas. En transición estratigráfica gradual se sitúan dolomías negras con microfósiles marinos (foraminíferos y algas) del Triásico. La sucesión se completa con brechas dolomíticas discordantes que, localmente, fosilizan el contacto entre la UME y los terrenos alpujárrides subyacentes y que son cabalgadas por las otras unidades tectónicas maláguides.

\subsubsection{Unidad Maláguide Inferior (UMI)}

Está formada por una sucesión pizarroso-grauváquica bien desarrollada y bastante clásica del Paleozoico Maláguide, que incluye a la Fm. Santi Petri como unidad estratigráfica más característica. No obstante, la presencia de los niveles típicos de calizas alabeadas fluctúa considerablemente en el área estudiada y, en algunas transversales, estas facies desaparecen o están muy adelgazadas por causas tectónicas. Por otra parte, las calizas alabeadas disminuyen hasta desaparecer hacia la parte alta de la formación, donde las pelitas oliváceas son la litología predominante. Sobre la Fm. Santi Petri aparece, muy localmente, la Fm. Falcoña, con sus dos miembros. La Fm. Almogía de la UMI muestra normalmente facies finas y su miembro areniscosoconglomerático inferior (Miembro Retamares) aparece solo localmente en la zona estudiada. Por ello, cuando no se observan las litofacies típicas de la Fm. Falcoña puede ser muy difícil distinguir la Fm. Almogía de las pelitas que caracterizan la parte alta de la Fm. Santi Petri. Finalmente, en discordancia sobre el Paleozoico de esta unidad aparece la Fm. Saladilla, que no está afectada por el metamorfismo alpino ni muestra a su techo el paquete de dolomías triásicas oscuras que se observa en la UME. Compuesta sobre todo por un gran paquete de arcillas y areniscas de color rojo, esta formación incluye niveles conglomeráticos de base canalizada en el tramo inferior de la sucesión y en los afloramientos más orientales del área estudiada, no así en su sector central.

\subsubsection{Unidad Maláguide Superior (UMS)}

Esta unidad es la mejor representada en la ZCV y en ella se centra el estudio realizado. Está integrada por materiales de edades que abarcan desde el Paleozoico hasta el Eoceno (Fig. 3). Los más antiguos, que denominaremos informalmente sucesión pre-Falcoña, están constituidos por una alternancia, bien estratificada en estratos medios a finos, de pizarras y areniscas cuarcíticas de grano muy fino (Figs. 4a-b), bien cementadas, entre las que aparecen lentejones carbonatados calcareníticos laminados y calcilutíticos (Fig. 4c) en los cuales se han hallado conodontos y dacrioconáridos del Devónico Inferior y Superior (Navas-Parejo et al., 2011, 2015; Navas-Parejo, 2012). Esta sucesión también incluye rocas volcanoclásticas, que se mencionan por vez primera en este trabajo dentro del Complejo Maláguide de la ZCV (Fig. 4d). Forman estratos de grosor centi- a decimétrico y textura areniscosa (Fig. 4b), con clastos de vidrio volcánico con vacuolas y fragmentos de feldespatos y minerales máficos muy alterados; localmente aparecen agrupados formando paquetes estratoides de espesor métrico a decamétrico, que se acuñan lateralmente pero que pueden alcanzar varios cientos de metros de continuidad lateral. En este último caso las rocas volcanoclásticas muestran texturas microbréchicas y areníticas fuertemente aplastadas y aparecen relacionadas con pequeños cuerpos de rocas magmáticas básicas con texturas afaníticas y faneríticas, con cristales de plagioclasa $\mathrm{y}$ anfíbol bien visibles, aunque por lo general muy alterados. 


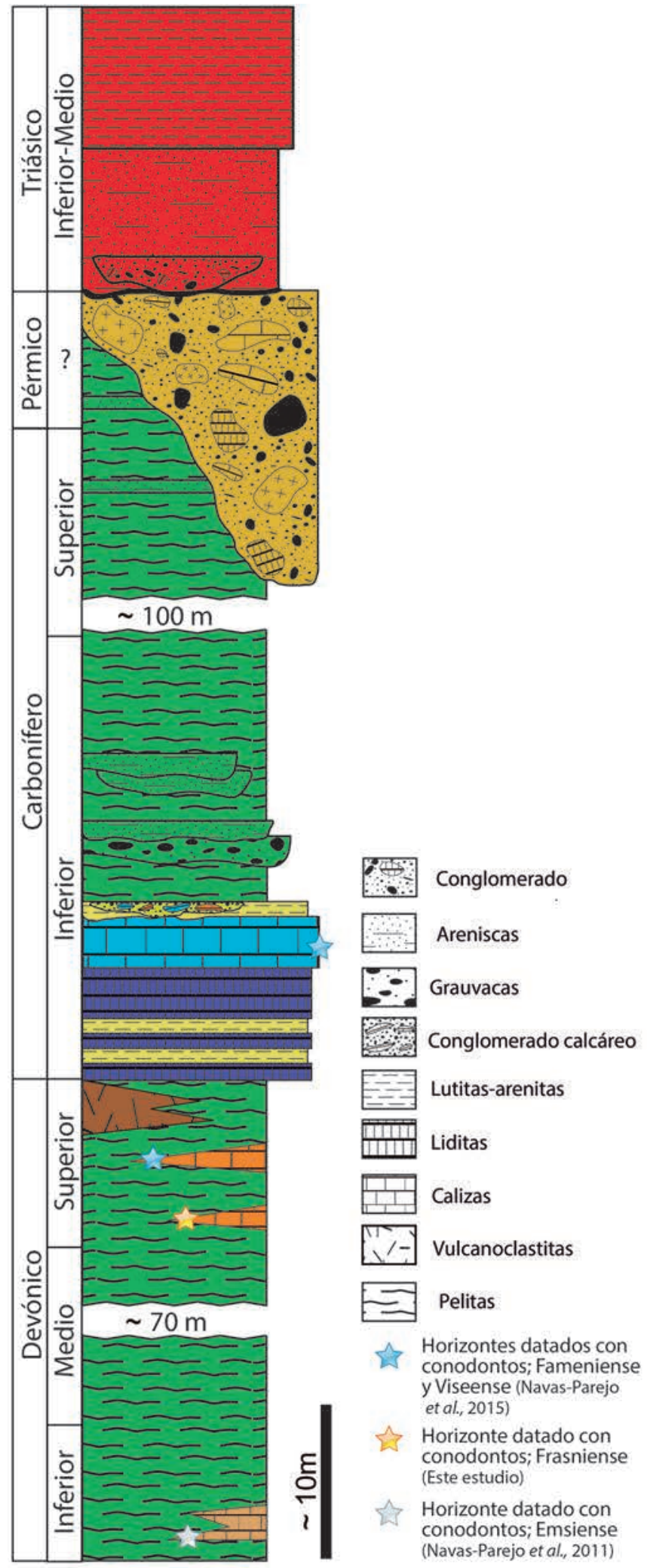

Figura 3. Sucesión estratigráfica sintética de la Unidad Maláguide Superior de la zona de Cogollos Vega.
Sobre los materiales de la sucesión pre-Falcoña reposa en concordancia la Fm. Falcoña, que aparece en numerosos puntos constituida por su miembro inferior de liditas y por su miembro superior de calizas. En la UMS ambos miembros están finamente estratificados y, en ocasiones, sus litologías más típicas alternan con delgados niveles de pizarras. Las calizas de esta formación pueden incluir nódulos de sílex. Esta formación es siempre muy poco potente, no más de 8-10 metros de espesor total en los mejores afloramientos. A menudo aparece intensísimamente plegada y, por causas tectónicas, su continuidad lateral raramente supera unos metros o, a lo sumo, unas decenas de metros. Sin embargo, pequeños testimonios muy laminados tectónicamente de sus rocas más típicas, las liditas, reaparecen continuamente en numerosos afloramientos ocupando siempre una posición estratigráfica y tectónica equivalente. Trabajos previos dentro de la ZCV han establecido la edad Viseense de las calizas de esta formación en algunos cortes (Navas-Parejo et al., 2015).

La Fm. Almogía de la UMS está compuesta mayoritariamente por una alternancia de pizarras, grauvacas y arenas, semejante a la ya descrita para la UMI. Sin embargo, en la parte estratigráficamente más baja de esta formación en el área estudiada de la UMS, afloran frecuentemente paquetes de grauvacas de tamaño de grano medio a muy grueso y en estratos gruesos, a menudo con niveles de conglomerados (Miembro Retamares). Hacia su parte más alta aparecen unas facies más finas y, sobre ellas, separadas por un contacto erosivo, descansa la Fm. Marbella. En los afloramientos estudiados (sector del Despeñadero-Cortijo de la Ermita) la Fm. Marbella se inicia con niveles pizarroso-grauváquicos de color pardoamarillento con pasadas de areniscas calcáreas que, a techo,

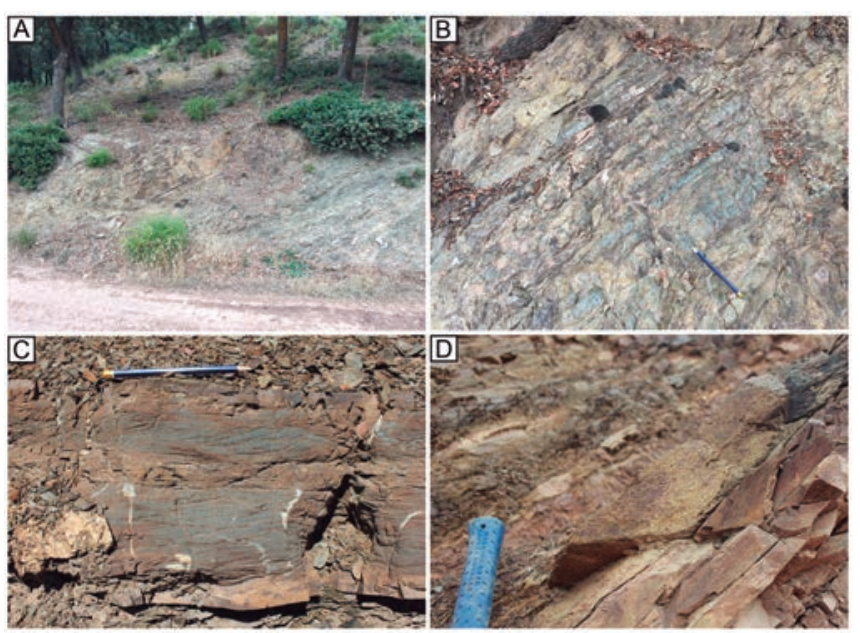

Figura 4. Aspecto de campo de la sucesión pre-Falcoña. a) Pelitas y arenitas finamente estratificadas con niveles volcanoclásticos. b) Arenitas finamente estratificadas. c) Calcarenitas con laminación cruzada. d) Detalle de un nivel volcanoclástico. 
pasan rápidamente a un conglomerado desorganizado con las características ya descritas (Herbig, 1984).

La Fm. Saladilla (Triásico) de la UMS incluye también conglomerados con bases canalizadas, intercalados entre areniscas y lutitas en el sector central del área estudiada. Destacan unos niveles de areniscas blancas con estratificación paralela a techo de esta formación en el sector del Cortijo del Mojino. Los materiales del Jurásico, Cretácico y Paleógeno se encuentran representados en la zona NE del área de estudio, entre los Cortijos del Mojino y de la Solana, formando una banda de bastante continuidad, pero de poca potencia e intensamente tectonizada. Están representados principalmente por unas dolomías blancas del Jurásico Inferior (Navarro-Vilá \& García-Dueñas, 1979). El resto de estos materiales más modernos aparecen en afloramientos dispersos y no se encuentran claramente in situ sino parcialmente removilizados en suelos y derrubios, si bien muestran facies típicas, equivalentes a las que se reconocen en las sucesiones de cobertera postpaleozoica de otros sectores maláguides (Martín-Algarra et al., 2004, entre otros).

\subsection{Afloramiento estudiado}

El afloramiento estudiado se ubica al $\mathrm{N}$ de la Umbría de Carboneras (coordenadas: $37^{\circ} 18^{\prime} 34$ " N, $3^{\circ} 45^{\prime} 48^{\prime \prime}$ $\mathrm{O})$, aproximadamente un kilómetro al $\mathrm{N}$ de la autovía A-92 a su paso por las cercanías de la antigua Venta del Molinillo (Fig. 2). La sección estudiada está compuesta por pelitas oliváceas de grano muy fino a fino, parcialmente recubiertas por suelos y derrubios superficiales, entre las que se intercalan delgados niveles más duros, areniscosos y carbonatados. La dirección promedio de la estratificación es $\mathrm{N} 60^{\circ}-70^{\circ} \mathrm{E}$ con buzamientos hacia el $\mathrm{N}$ tanto normales como invertidos. La polaridad estratigráfica en distintos puntos se reconoce gracias a la presencia local de estructuras sedimentarias en los estratos más duros: laminación cruzada y paralela, bases netas, tránsitos litológicos graduales hacia techo desde granulometrías más gruesas (arena media a fina generalmente) a otras progresivamente más finas. Localmente se observan secuencias propias de turbiditas distales.

El lentejón calizo del que procede la muestra 17A67 (Figs. 5a-b) tiene 40 centímetros de potencia máxima y unos 4 metros de continuidad lateral, con dirección y buzamiento $\mathrm{N} 90^{\circ} \mathrm{E} / 65^{\circ} \mathrm{N}$. El estrato, de color gris oscuro, muestra una textura calcarenítica laminada (Fig. 6a), con tamaño de grano de arena gruesa a media (Fig. 6b) y lateralmente se divide en tres niveles separados por intercalaciones pelíticas. Su contacto con las pelitas suprayacentes es una superficie neta (Figs. 6a-b) con estructuras de erosión por corrientes (scour marks; Fig. 6c) mientras que, hacia su parte inferior, disminuye gradualmente el tamaño de grano en tránsito progresivo a las pelitas subyacentes. Todo ello indica que el estrato está invertido en este punto, lo que explica la presencia, unos metros al $\mathrm{S}$ del mismo y por debajo, de un pequeño afloramiento de liditas atribuibles al miembro inferior de la Fm. Falcoña. Intensamente replegadas (Fig. 5c), estas liditas seguramente constituyen el núcleo de un pequeño sinclinal muy apretado vergente al SE. No obstante, hacia el $\mathrm{N}$ de este afloramiento, la polaridad normal de la sucesión se recupera en una banda bien estratificada de liditas sobre la que aparecen pequeños bloques dispersos de calizas, litologías ambas atribuibles a la Fm. Falcoña.

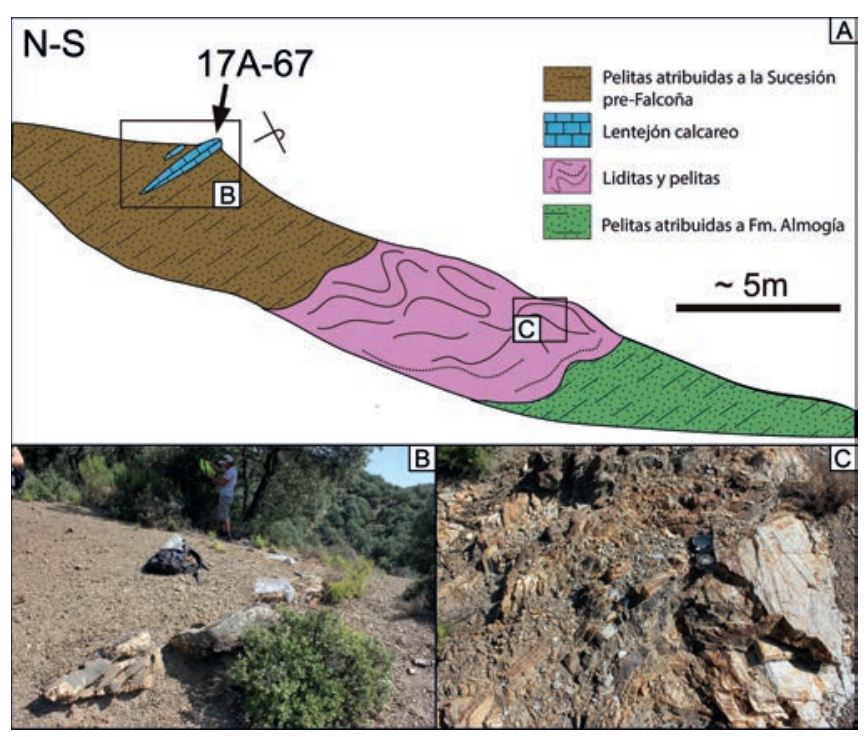

Figura 5. a) Esquema geológico del afloramiento estudiado en el área de Umbría de Carboneras, con indicación de la localización de las imágenes b y c y de la muestra 17A-67. b) Lentejón calcáreo del Frasniense. c) Liditas intensamente replegadas.

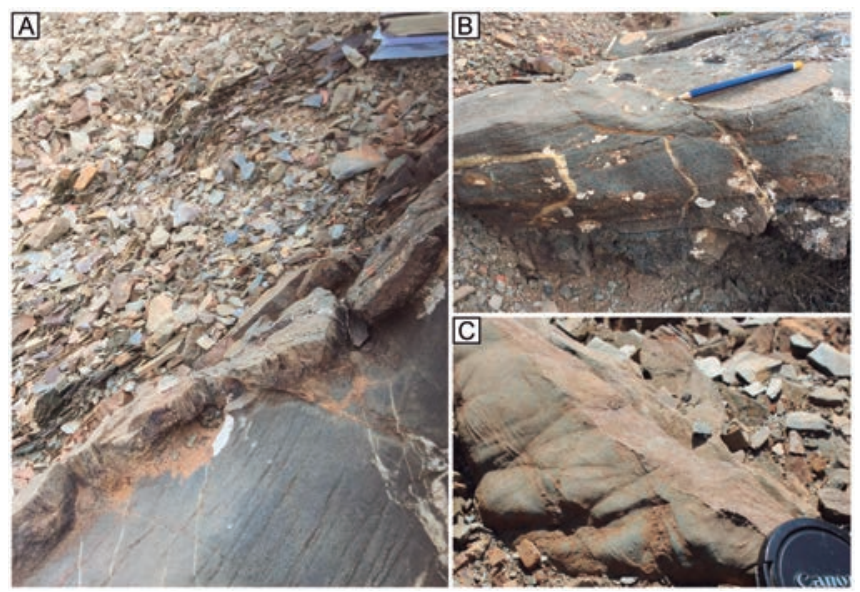

Figura 6. Detalles de la textura y estructura del nivel estudiado. a) Contacto con las pelitas geométricamente suprayacentes. b) Textura arenítica laminada y paso a las pelitas subyacentes. c) Huellas de abrasión por corrientes en la superficie geométrica superior del estrato (muro invertido). 


\section{PALEONTOLOGÍA}

\subsection{Material y métodos}

Se ha disuelto 1 kilogramo de la muestra 17A-67, tomada en el nivel calizo anteriormente descrito. La muestra ha proporcionado una abundante fauna de conodontos, en concreto, en $1 \mathrm{~kg}$ de muestra se han obtenido 761 elementos conodontales pectiniformes. Para la disolución de la muestra se ha seguido el procedimiento propuesto por Jeppsson et al. (1999). Los ejemplares con mejor conservación se han fotografiado mediante microscopio electrónico de barrido (Figs. 7-8). La Tabla 1 muestra su distribución bioestratigráfica, las biozonas donde aparecen y el número de elementos hallados de cada especie.
El estado de conservación de los ejemplares no es óptimo, ya que la mayoría de éstos se encuentran fragmentados y con numerosas fracturas. No obstante, los rasgos diagnósticos de muchos ejemplares completos se encuentran suficientemente bien preservados para su identificación. El CAI (Color Alteration Index: Epstein et al., 1977) de los elementos es 5. Para su determinación se ha utilizado una escala de CAI estándar, así como tablas Munsell, según la escala de color que establecieron Epstein et al. (1977) y Rejebian et al. (1987).

Los ejemplares (con siglas 17A-67) están depositados en el museo del Departamento de Estratigrafía y Paleontología de la Universidad de Granada.

Tabla 1. Biofacies y biozonas presentes en la muestra 17A-67. La distribución bioestratigráfica de los géneros y especies de conodontos atiende a la establecida por diversos autores. Dado que no existe acuerdo en dicha distribución, tal como se comenta en el apartado de sistemática, en este esquema se ha optado por representar la distribución más amplia posible. El esquema de la biozonación de la columna izquierda está basado en Sandberg et al. (1989) y Ziegler \& Sandberg (1990) para el Frasniense, y en Klapper \& Johnson (1990) para el Givetiense; el de la columna derecha corresponde al establecido por Klapper (1989) y Klapper \& Becker (1998, 1999), ambas recogidas por Klapper \& Kirchgasser (2016).

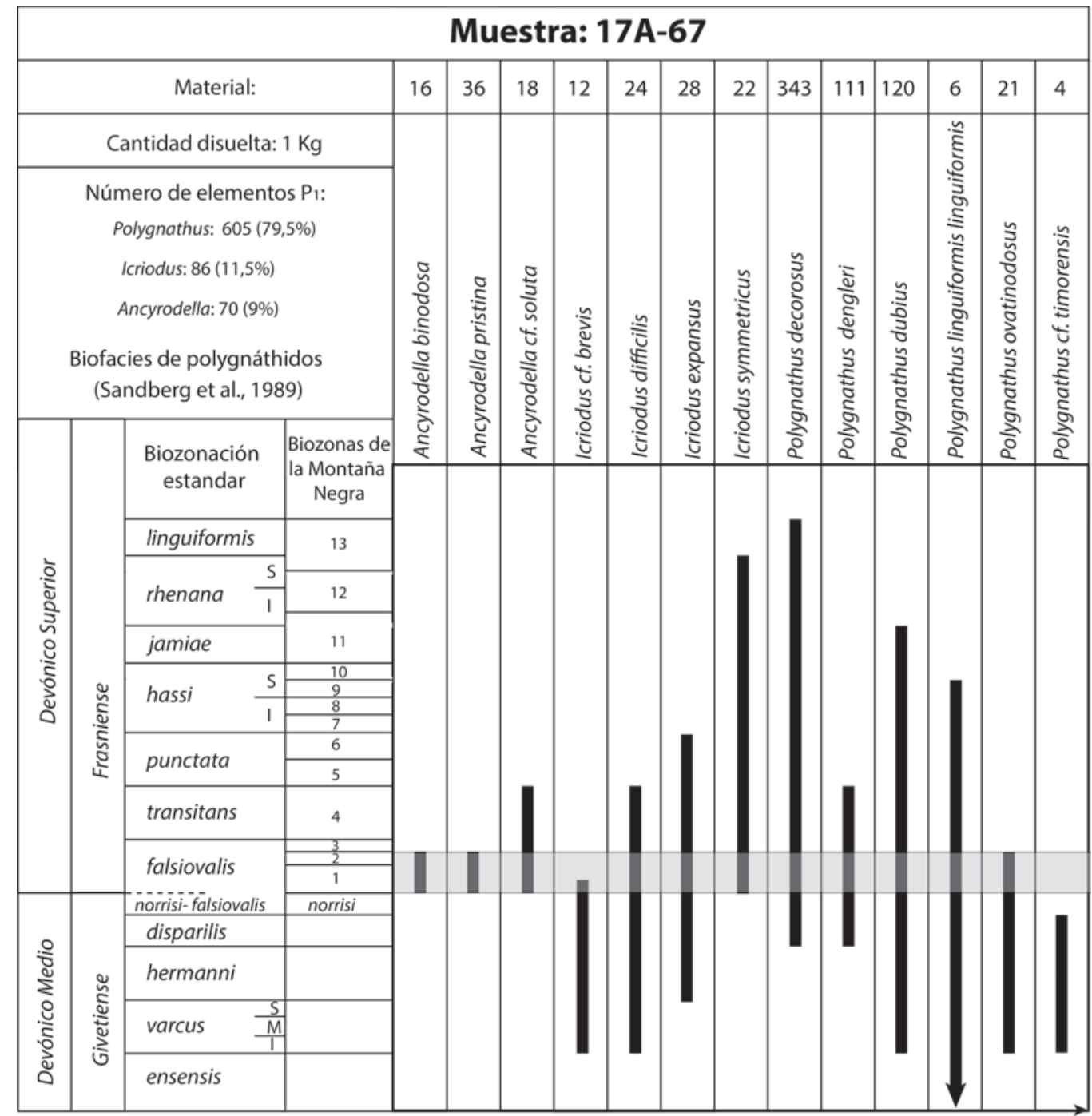




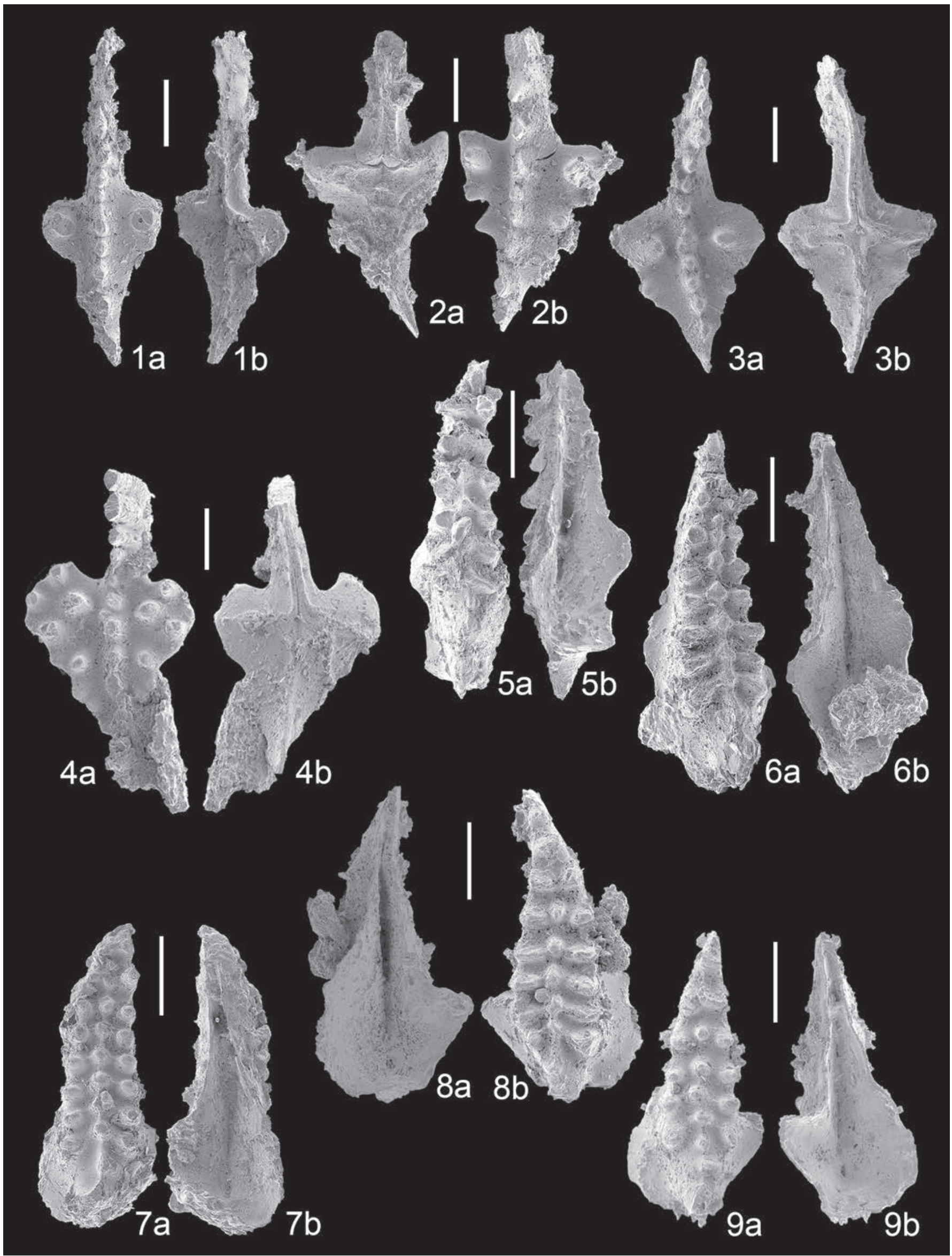




\subsection{Bioestratigrafía}

En la muestra 17A-67 se han identificado tres géneros: Ancyrodella, Icriodus y Polygnathus, y un total de trece especies (Tabla 1): Ancyrodella binodosa Uyeno, 1967, Ancyrodella pristina Khalymbadzha \& Chernysheva, 1970, Ancyrodella cf. soluta Sandberg, Ziegler \& Bultynck, 1989, Icriodus cf. brevis Stauffer, 1940, Icriodus difficilis Ziegler \& Klapper, 1976, Icriodus expansus Branson \& Mehl, 1934, Icriodus symmetricus Branson \& Mehl, 1934, Polygnathus decorosus Stauffer, 1938, Polygnathus dengleri Bischoff \& Ziegler, 1957, Polygnathus dubius Miller, 1889, Polygnathus linguiformis linguiformis Hinde, 1879, Polygnathus ovatinodosus Ziegler \& Klapper, 1976 (en Ziegler et al., 1976) y Polygnathus cf. timorensis Klapper, Philip \& Jackson, 1970.

Los afloramientos del Complejo Maláguide generalmente adolecen de continuidad y, en el afloramiento estudiado, no es posible levantar una sección estratigráfica detallada en la que se pueda establecer una bioestratigrafía precisa, ni realizar ninguna estimación ni aportación bien fundamentada sobre la distribución biostratigráfica de las diferentes especies halladas. Por ello, en este trabajo se aceptan las distribuciones bioestratigráficas establecidas por diferentes autores aunque, dado que no siempre existe acuerdo sobre ellas, en la Tabla 1 se reflejan las distribuciones más amplias propuestas.

La presencia de Ancyrodella pristina y Ancyrodella binodosa sitúan al nivel carbonatado en el intervalo comprendido entre Zona MN1 y MN2 de la Montaña Negra (Klapper, 1989). Las especies Ancyrodella cf. soluta, Icriodus cf. brevis, Icriodus expansus, Icriodus symmetricus, Polygnathus decorosus, Polygnathus dengleri, Polygnathus dubius, Polygnathus linguiformis linguiformis y Polygnathus ovatinodosus coexisten en las citadas zonas. Sin embargo, Icriodus difficilis parece que no coexiste ya que las referencias consultadas estiman que solo alcanza hasta la Zona de norrisi; Polygnathus cf. timorensis no es una especie Frasniense, no habiéndose mencionado su existencia más allá de la Zona de disparilis. Se ignora si Icriodus difficilis pudiere haber tenido, o no, una mayor extensión y alcanzar la Zona MN1 en el área de estudio. No obstante, en todo caso y habida cuenta de las características sedimentológicas del horizonte estudiado, ciertamente los ejemplares de Polygnathus $\mathrm{cf}$. timorensis fueron resedimentados tras ser erosionados de niveles algo más antiguos junto con otros conodontos más o menos contemporáneos de la sedimentación autóctona entre la que se intercala el nivel calcarenítico datado.

\subsection{Biofacies}

Aunque la fauna estudiada procede de una sola muestra y únicamente se ha disuelto 1 kilogramo de la misma, la extraordinaria abundancia de elementos conodontales permite hacer una primera aproximación a la biofacies presente en estos sedimentos. El número de elementos $\mathrm{P}_{1}$ de Polygnathus encontrados en la muestra es de 605 (siguiendo a Sandberg et al., 1989, se han incluido en este cálculo los elementos $\mathrm{P}_{1}$ de Polygnathus dengleri), el de Icriodus es de 86 y el de Ancyrodella es de 70. Así pues, Polygnathus representa un 79,5\%, Icriodus un $11,5 \%$ y Ancyrodella un 9\%.

Sandberg et al. (1989) reconocieron, en las zonas de falsiovalis y transitans, cinco biofacies ordenadas según ambientes sedimentarios situados de mayor a menor profundidad y en dirección a la línea de costa: i) mesotáxidos-polygnáthidos, ii) polygnáthidos-icriódidos, iii) polygnáthidos-ancyrodéllidos, iv) polygnáthidos y v) pandorinellínidos. De acuerdo con estos autores, la fauna de la muestra estudiada se corresponde con una biofacies de polygnáthidos.

\subsection{Sistemática}

En el apartado taxonómico se ha seguido la clasificación establecida por Sweet (1988) y Sweet \& Donoghue (2001). En la descripción de los elementos conodontales se ha usado la nomenclatura propuesta por Sweet (1988) y García-López (1984), así como las modificaciones en la terminología sobre la orientación de los elementos con los términos dorsal, ventral, rostral, caudal, oral y aboral, introducidos por Purnell et al. (2000). Para la identificación de las especies se ha utilizado únicamente el elemento $\mathrm{P}_{1}$.

Filo CHORDATA Bateson, 1886

Clase CONODONTA Pander, 1856

Subclase CONODONTI Branson, 1938

Orden OZARKODINIDA Dzik, 1976

Familia Spathognathodontidae Hass, 1959

Figura 7. 1) Elemento $\mathrm{P}_{1} 17 \mathrm{~A} 67-3$ de Ancyrodella binodosa. Vista oral (a) y aboral (b). 2) Elemento $\mathrm{P}_{1}$ 17A67-17 de Ancyrodella pristina. Vista oral (a) y aboral (b). 3) Elemento P 17A67-12 de Ancyrodella pristina. Vista oral (a) y aboral (b). 4) Elemento $\mathrm{P}_{1}$ 17A67-2 de Ancyrodella cf. soluta. Vista oral (a) y aboral (b). 5) Elemento $\mathrm{P}_{1}$ 17A67-10 de Icriodus cf. brevis. Vista oral (a) y aboral (b). 6) Elemento $\mathrm{P}_{1} 17 \mathrm{~A} 67-8$ de Icriodus expansus. Vista oral (a) y aboral (b). 7) Elemento $\mathrm{P}_{1} 17 \mathrm{~A} 67-21$ de Icriodus symetricus. Vista oral (a) y aboral (b). 8) Elemento $\mathrm{P}_{1} 17 \mathrm{~A} 67-7$ de Icriodus difficilis. Vista aboral (a) y oral (b). 9) Elemento $\mathrm{P}_{1}$ 17A67-1 de Icriodus difficilis. Vista oral (a) y aboral (b). Escala $=200 \mu \mathrm{m}$. 


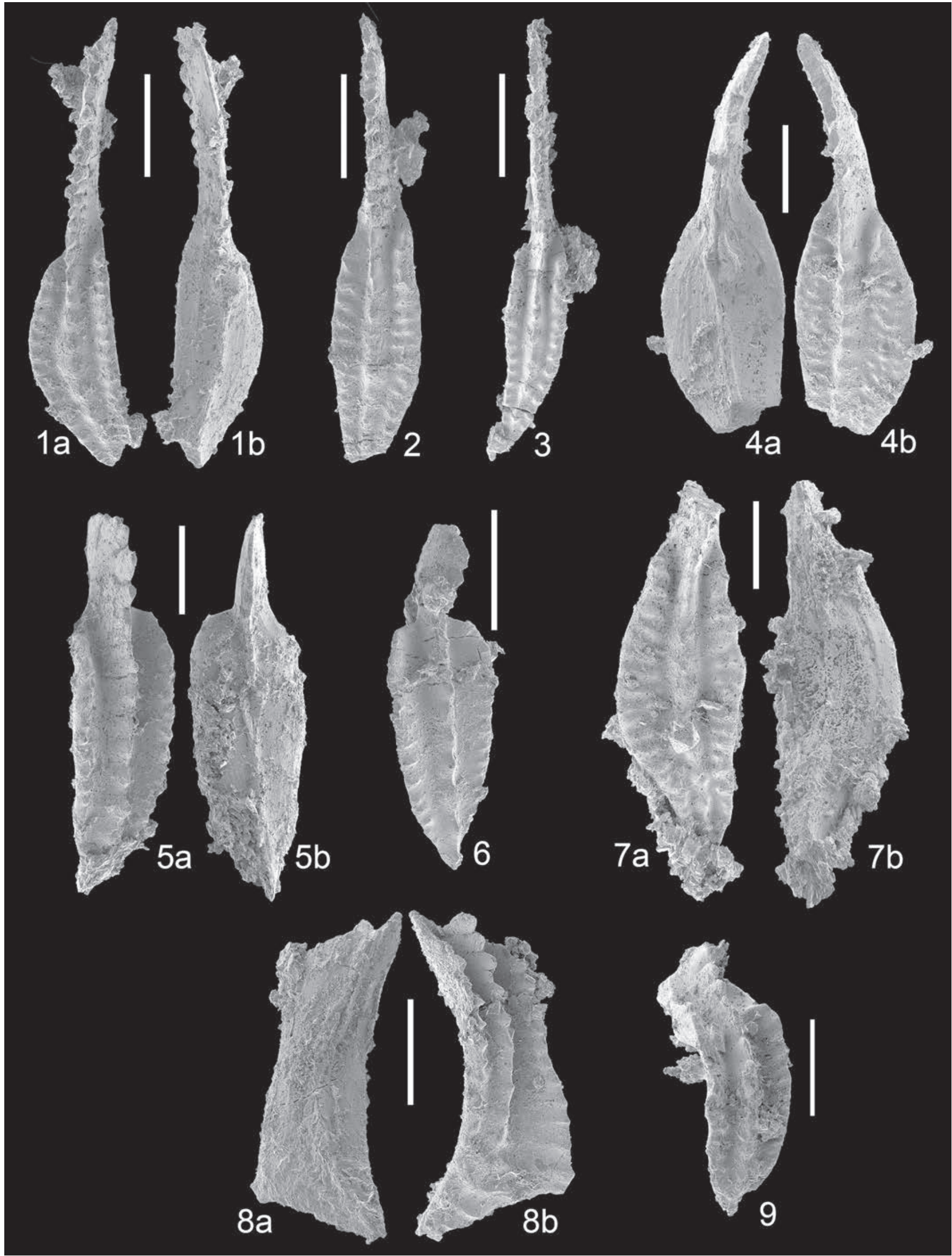


Género Ancyrodella Ulrich \& Bassler, 1926

Especie tipo Ancyrodella nodosa Ulrich \& Bassler, 1926

Ancyrodella binodosa Uyeno, 1967

(Figs. 7.1a-b)

*1967 Ancyrodella rotundiloba binodosa n. subsp.; Uyeno, p. 4, Pl. 1, Figs. 2, 4-5.

1976 Ancyrodella rotundiloba binodosa; García-López, Pl. 2, Fig. 1

1982 Ancyrodella rotundiloba binodosa; Morzadec \& Weyant, p. 30, Pl. 4, Figs. 1-3, 4-6.

1982 Ancyrodella binodosa; Mouravieff, Pl. 1, Figs. $1-21$.

1987 Ancyrodella binodosa; García-López, Pl. 1, Figs. $1-5$.

1989 Ancyrodella binodosa; Sandberg, et al., p. 210, Pl. 1, Figs. 3-4, 9-10, 13-14.

1999 Ancyrodella binodosa; Lazreq, p. 57, Pl. 3, Figs. 4-7.

2000 Ancyrodella binodosa; Çapkinoglu \& Gedik, Pl. 5, Figs. 5-6.

2008 Ancyrodella binodosa; Ovnatanova \& Kononova, p. 1080, Pl. 26, Figs. 1-2.

2013 Ancyrodella binodosa; Gholamalian et al., Pl. 1, Figs. 14-19.

2013 Ancyrodella binodosa; Gouwy et al., p. 8, Figs. 6 F-G.

2017 Ancyrodella binodosa; Ovnatanova et al., p. 1045, Pl. 8, Fig. 8.

Número de elementos P1. 16 ejemplares.

Descripción. El elemento $\mathrm{P}_{1}$ posee una plataforma subtriangular muy estrecha, con dos lóbulos laterales de contornos redondeados situados en su parte ventral y con un extremo dorsal muy puntiagudo. Cada lóbulo lateral presenta un gran nódulo en la superficie oral. La carena recorre toda la plataforma y está constituida por nódulos redondeados, siendo más grandes y espaciados hacia su parte dorsal. La lámina libre es de longitud similar a la plataforma y está adornada con dentículos que tienen mayor altura en su extremo ventral. Su cavidad basal es cruciforme y grande, y las quillas apenas están desarrolladas, pero sí presenta surcos someros.

Observaciones. Ancyrodella binodosa se diferencia de Ancyrodella pristina en que: i) su ornamentación consiste en dos grandes nódulos en sus lóbulos laterales, careciendo de los pequeños que ornamentan el borde de la plataforma de esta última; ii) el contorno de su plataforma no es tan claramente triangular como en A. pristina; y iii) la lámina libre tiene la misma longitud de la plataforma, mientras que en A. pristina es ligeramente más corta.

Distribución bioestratigráfica. Parte media de la Zona inferior de falsiovalis (Devónico Superior, Sandberg et al., 1989); Zona MN1 - Zona MN2 de la Montaña Negra (Ovnatanova et al., 2017).

$$
\begin{gathered}
\text { Ancyrodella pristina Khalymbadzha \& Chernysheva, } \\
1970
\end{gathered}
$$

(Figs. 7.2a-b, 7.3a-b)

*1970 Ancyrodella pristina n. sp.; Khalymbadzha \& Chernysheva, p. 89, Pl. 1, Figs. 5, 7.

1993 Ancyrodella pristina; Ji \& Ziegler, p. 53, P1. 1, Figs. 7-9.

1999 Ancyrodella pristina; Lazreq, p. 60, Pl. 3, Figs. $8,9,13$.

2007 Ancyrodella rotundiloba pristina; Aboussalam \& Becker, p. 364, Figs. 9 K, L, O-R.

2007 Ancyrodella pristina; Miller, Figs. 2 A-Y.

2008 Ancyrodella pristina; Liao \& Valenzuela-Ríos, p. 2, Figs. 6 I-J.

2014 Ancyrodella pristina; Bahrami et al., Pl. 1, Fig. 17.

2016 Ancyrodella rotundiloba pristina; Klapper \& Kirchgasser, p. 537, Figs. 7.5, 7.10.

2017 Ancyrodella pristina; Ovnatanova et al., p. 1049, Pl. 11, Fig. 7.

Figura 8. 1) Elemento $P_{1} 17$ A67-5 de Polygnathus decorosus. Vista oral (a) y aboral (b). 2) Elemento $\mathrm{P}_{1}$ 17A67-48 de Polygnathus decorosus. Vista oral. 3) Elemento $\mathrm{P}_{1}$ 17A67-45 de Polygnathus decorosus. Vista oral. 4) Elemento $\mathrm{P}_{1}$ 17A67-25 de Polygnathus ovatinodosus. Vista aboral (a) y oral (b). 5) Elemento $P_{1} 17 \mathrm{~A} 67-31$ de Polygnathus dengleri. Vista oral (a) y aboral (b). 6) Elemento $\mathrm{P}_{1}$ 17A67-41 de Polygnathus dengleri. Vista oral. 7) Elemento $\mathrm{P}_{1}$ 17A67-28 de Polygnathus dubius. Vista oral (a) y aboral (b). 8) Elemento $\mathrm{P}_{1}$ 17A67-22 de Polygnathus linguiformis linguiformis. Vista aboral (a) y oral (b). 9) Elemento $\mathrm{P}_{1}$ 17A67-27 de Polygnathus timorensis. Vista oral. Escala $=300 \mu \mathrm{m}$. 
Número de elementos P1. 36 ejemplares.

Descripción. El elemento $\mathrm{P}_{1}$ consta de una plataforma de contorno claramente triangular, con los márgenes ventrales rectos o redondeados y ornamentada con dos nódulos grandes situados a ambos lados de la plataforma en su parte ventral, y una serie de nódulos de pequeño a mediano tamaño localizados a lo largo de los bordes de la plataforma alcanzando su extremo dorsal. La relación longitudinal entre la lámina y la plataforma es de $1 / 3$ de lámina libre y $2 / 3$ de plataforma. La cavidad basal es cruciforme y tiene un tamaño grande en comparación con el del elemento. Las quillas secundarias, poco desarrolladas, parten de la cavidad basal y alcanzan la parte central de sus lóbulos.

Observaciones. Esta especie es similar a Ancyrodella rotundiloba, pero su plataforma es más claramente triangular y tiene una ornamentación más simple; asimismo, su cavidad basal es más grande. Véanse también, en Ancyrodella binodosa, las diferencias con esta otra especie.

Distribución bioestratigráfica. Zona $\mathrm{MN} 1$ de la Montaña Negra (Aboussalam \& Becker, 2007); Zona MN1 y Zona MN2 de la Montaña Negra (Liao \& ValenzuelaRíos, 2008; Ovnatanova et al., 2017).

Ancyrodella cf. soluta Sandberg, Ziegler \& Bultynck, 1989

(Figs. 7.4a-b)

*1989 Ancyrodella soluta n. sp.; Sandberg et al., p. 211, Pl. 1, Figs. 5-6, 11-12; Pl. 2, Figs. 1-4.

1993 Ancyrodella soluta; Racki \& Bultynck, Pl. 6, Figs. 2-3, 7; Pl. 7, Figs. 1-3, 9-11.

1999 Ancyrodella soluta; Lazreq, p. 60, P1. 3, Fig. 10.

2008 Ancyrodella soluta; Liao \& Valenzuela-Ríos, p.7, P1. 6, Figs. K-P.

2017 Ancyrodella soluta; Ovnatanova et al., p. 1055, Pl. 5, Fig. 8; Pl. 23, Figs. 1, 4-6; Pl. 29, Fig. 6; Pl. 31, Fig. 1.

Número de elementos P1. 18 ejemplares.

Descripción. El elemento $\mathrm{P}_{1}$ se caracteriza por tener una plataforma con contornos redondeados en los bordes ventrales y puntiaguda en su parte dorsal, y está ornamentada con varios nódulos de diferente tamaño distribuidos sobre su superficie, destacando dos grandes nódulos sobre sus lóbulos laterales. Su cavidad basal es cruciforme y de tamaño mediano; de ella parten dos pequeñas quillas secundarias presentando un desarrollo incipiente del surco ventral y de la quilla dorsal.
Observaciones. Ancyrodella soluta es una forma intermedia entre A. pristina and A. rotundiloba. Se diferencia de A. pristina por poseer una mayor profusión de nódulos sobre su plataforma y tener una cavidad basal de menor tamaño. No obstante, Kralick (1994) y Klapper (2000), argumentan que estos criterios no son suficientemente sólidos como para establecer una nueva especie. Los ejemplares aquí hallados difieren del holotipo de Sandberg et al. (1989) en que poseen una ornamentación más simple, con nódulos menos numerosos. No obstante, se han incluido en esta especie por considerar que no caen en el intervalo de variabilidad de $A$. pristina, la cual desarrolla nódulos solo sobre el borde de la plataforma.

Distribución bioestratigráfica. Parte media de la Zona inferior de falsiovalis - Zona de transitans (Sandberg et al., 1989); Zonas MN2 - MN4 de la Montaña Negra (Ovnatanova et al., 2017).

Familia Icriodontidae Müller \& Müller, 1957

Género Icriodus Branson \& Mehl, 1934

Especie tipo Icriodus expansus Branson \& Mehl, 1934.

Icriodus cf. brevis Stauffer, 1940

(Figs. 7.5a-b)

*1940 Icriodus brevis n.sp.; Stauffer, p. 424, P1. 60, Figs. 36, 43, 44, 52.

1976 Icriodus brevis; Ziegler et al., p. 117, Pl. 1, Figs. 10-16.

1977 Icriodus brevis; Weddige, p. 285, Pl. 2, Fig. 37.

1982 Icriodus brevis; Morzadec \& Weyant, p. 32, Pl. 1, Figs. 23-24.

1987 Icriodus brevis; Bultynck, p. 158, Pl. 6, Figs. $1-14$.

1989 Icriodus brevis; Mawson \& Talent, Pl. 1, Figs. 16-19.

1999 Icriodus brevis; Lazreq, p. 64, Pl. 1, Figs. 15-16.

1999 Icriodus brevis; Talent et al., Pl. 4, Figs. 1-2.

2001 Icriodus brevis; Liao et al., p. 17, P1. 2, Figs. 3-4.

2002 Icriodus brevis; Draganits et al., Pl. 1, Figs. 15-18.

2003 Icriodus brevis brevis; Aboussalam, p. 164, Pl. 23, Figs. 3-4.

2010 Icriodus brevis; Narkiewicz \& Bultynck, Fig. 13.10-13.11; Fig. 18.17.

Número de elementos P1. 12 ejemplares. 
Descripción. El elemento $\mathrm{P}_{1}$ se caracteriza por tener un huso estrecho y ligeramente biconvexo. Los dentículos del huso tienen una sección de redondeada a oval y los siete de su fila central se disponen alternando con los seis de las filas laterales. La fila central de los dentículos se prolonga dorsalmente mucho más que las filas laterales, describiendo en vista lateral una forma de abanico. Su cavidad basal se ensancha a partir de su mitad dorsal y en su lado caudal desarrolla un espolón muy sutil y redondeado. En vista lateral su borde dorsal es puntiagudo.

Observaciones. Los ejemplares hallados presentan un deficiente estado de conservación por lo que se incluyen en esta especie con reservas. Véanse también las observaciones de Icriodus difficilis.

Distribución bioestratigráfica. Zona de varcus inferior (rhenanus) - parte superior de la Zona inferior de falsiovalis (Bultynck, 2003); Zona de rhenanus - parte más baja de la Zona MN1 (Liao \& Valenzuela-Ríos, 2008).

\section{Icriodus expansus Branson \& Mehl, 1938}

(Fig. 7.6a-b)

*1938 Icriodus expansus n.sp.; Branson \& Mehl, p. 160, Pl. 26, Figs. 18-19.

1982 Icriodus expansus; Morzadec \& Weyant, p. 32, Pl.1, Figs. 12-16.

1987 Icriodus expansus; García-López, p. 76, P1. 9, Figs.7-22.

1978 Icriodus expansus; Orchard, p. 928, Pl. 109, Figs. 2-5, 7, 10, 14, 15, 20, 23.

1989 Icriodus expansus; Mawson \& Talent, Pl.1, Figs. 11-12.

2002 Icriodus expansus; Draganits et al., Pl. 2, Fig.10.

2010 Icriodus expansus; Narkiewicz \& Bultynck, Figs. 13.14-18; Figs. 17.9-10; Figs. 18.7-9, 18.13-16.

2014 Icriodus expansus; Bahrami et al., Pl. 1, Figs. 36-38.

2017 Icriodus expansus; Ovnatanova et al., P1. 24, Figs. 3-4.

Número de elementos P1. 28 ejemplares.

Descripción. La forma del huso del elemento $\mathrm{P}_{1}$ es ligeramente biconvexa, alargada y su extremo dorsal es puntiagudo. Los dentículos de la carena presentan sección redondeada y los de las filas laterales son algo más ovalados, no encontrándose alineados con los de la carena. La carena se prolonga dorsalmente con dos o tres dentículos fusionados. Su cavidad basal es redondeada, siendo más ancha en su mitad dorsal.

Observaciones. Icriodus expansus no presenta espolón ni seno, características que lo diferencian de I. difficilis. La expansión de la cavidad basal en I. expansus es mayor que en $I$. difficilis.

Distribución bioestratigráfica. Zona inferior de hermanni - Zona de punctata (Narkiewicz \& Bultynck, 2010).

Icriodus symmetricus Branson \& Mehl, 1934

(Figs. 7.7a-b)

*1934 Icriodus symmetricus n.sp.; Branson \& Mehl, p. 226, Pl. 13, Figs. 1-3.

1978 Icriodus symmetricus; Orchard, Pl. 109, Figs. 17, 22, 23, 26, 32, 34.

1980 Icriodus symmetricus; Johnson et al., Pl. 3, Fig. 4.

1980 Icriodus symmetricus; Perri \& Spalletta, P1. 3, Figs. 4a-5c.

1982 Icriodus symmetricus; Morzadec \& Weyant, p. 33, P1.1, Figs. 3-5.

1985 Icriodus symmetricus; Klapper \& Lane, p. 921, Fig. 11.1.

1987 Icriodus symmetricus; García-López, p. 82, Pl. 10, Figs. 1-10.

1989 Icriodus symmetricus; Mawson \& Talent, Pl. 2, Figs. 4-5.

1992 Icriodus symmetricus; Carls \& Gong, Pl. 3, Figs. $18-21$.

1993 Icriodus symmetricus; Ji \& Ziegler, Pl. 5, Figs. $11-13$

1992 Icriodus symmetricus; Sandberg et al., Pl. 1, Fig. 3.

1999 Icriodus symmetricus; Lazreq, p. 65, Pl. 4, Figs. 12-13.

2003 Icriodus symmetricus; Aboussalam, p. 168, Pl. 23, Figs. 11-12.

2007 Icriodus symmetricus; Aboussalam \& Becker, Fig. 8 C.

2007 Icriodus symmetricus; Over, Pl. 10, Figs. 18-19.

2008 Icriodus symmetricus; Gholamalian \& Kebriaei, Pl. 1, Figs. 14-16.

2010 Icriodus symmetricus; Narkiewicz \& Bultynck, Figs. 15. 11-12. 
2011 Icriodus symmetricus; Narkiewicz \& Bultynck, Pl. 9, Fig. 8.

2012 Icriodus symmetricus; Liao \& Valenzuela-Ríos, p. 826, Figs. 6 A-J.

2014 Icriodus symmetricus; Rodríguez-Cañero \& Martín-Algarra, Fig. 10.4.

2015 Icriodus symmetricus; Mahboubi \& Gatovsky, Fig. 3 L, P.

2017 Icriodus symmetricus; Ovnatanova et al., Pl. 9, Fig. 9; P1. 17, Fig. 8; Pl. 25, Fig. 8; Pl. 31, Fig. 5; Pl. 34, Fig. 12.

Número de elementos P1. 22 ejemplares.

Descripción. El elemento $P_{1}$ es estrecho en relación con su longitud y ventralmente presenta una ligera flexión hacia el lado caudal. Las filas laterales de dentículos se disponen paralelamente a la central, excepto en su extremo ventral. Los dentículos de la fila central se encuentran conectados por puentes longitudinales. Lo más característico de este elemento es que dicha fila central de dentículos es más alta que las laterales en su extremo dorsal. Los dentículos de la fila media no están alineados con los de las filas laterales, estando algo mas adelantados con respecto a éstas. La cavidad basal es estrecha en su mitad ventral y se ensancha adoptando forma subcircular en su parte dorsal.

Observaciones. Icriodus symmetricus no presenta espolón ni seno, características que lo diferencian de $I$. difficilis. De I. expansus se distingue porque el huso no se expande dorsalmente y porque su fila central es más alta que las laterales en su parte dorsal.

Distribución bioestratigráfica. Zona MN2 - Zona MN13 (Narkiewicz \& Bultynck, 2010); Zona de falsiovalis (MN1) - Zona de rhenana superior (MN13) (Liao \& Valenzuela-Ríos, 2012).

Icriodus difficilis Ziegler \& Klapper, 1976

(Figs. 7.8a-b, 7.9a-b)

*1976 Icriodus difficilis n. sp.; Ziegler et al., p. 117, Pl.1, Figs. 1-7, 17.

1977 Icriodus difficilis; Weddige, Pl. 2, Fig. 36.

1978 Icriodus difficilis; Orchard, Pl. 109, Figs. 9, 12, $13,16,18$.

1987 Icriodus difficilis; Bultynck, Pl. 9, Figs. 25, 26.

1989 Icriodus difficilis; Mawson \& Talent, Pl. 1, Figs. 6-10.
1992 Icriodus difficilis; Bardashev, P1. 11, Figs. 5, 8, $11,16,17$.

1992 Icriodus difficilis; Carls \& Gong, Pl. 1, Figs. 16-17.

1999 Icriodus difficilis; Lazreq, p. 64, Pl.1, Figs. 18-19.

2001 Icriodus difficilis; Liao et al., p. 17, Pl. 1, Figs. 1-7.

2002 Icriodus difficilis; Draganits et al., P1. 1, Figs. 1-2, 6-8, 13.

2002 Icriodus difficilis; García-López et al., Pl. 2, Fig. 6.

2003 Icriodus difficilis; Aboussalam, p. 166, Pl. 23, Figs. 1-2.

2008 Icriodus difficilis; Liao \& Valenzuela-Ríos, p. 7, Fig. 4 C-D.

2010 Icriodus difficilis; Narkiewicz \& Bultynck, Fig. 17.11; Figs. 18.18 y 18.19; Fig. 19.13.

2012 Icriodus difficilis; Matyja, Fig. 17 D.

Número de elementos P1. 24 ejemplares.

Descripción. El elemento $P_{1}$ se caracteriza por presentar un espolón dirigido ventralmente y un seno moderadamente desarrollado en el margen caudal dorsal de la cavidad basal. La forma del huso varía de recta a ligeramente curvada y la carena se prolonga dorsalmente con dos o tres dentículos, siendo el último, o cúspide, de mayor tamaño. Las filas laterales se disponen aproximadamente paralelas a la carena. Los seis dentículos laterales poseen sección de redondeada a ovalada y pueden estar conectados con los nueve centrales, de sección mas redondeada, a través de pequeños puentes transversales. El espaciado entre dichos puentes es más amplio en la parte ventral que en la parte dorsal.

Observaciones. Icriodus difficilis se diferencia de $I$. expansus por desarrollar un espolón y un seno mucho más marcados. Se diferencia de I. brevis porque la extensión dorsal de la carena es más corta, tiene más dentículos en las filas laterales y éstos no tienden a alternar con los de la fila central.

Distribución bioestratigráfica. Zona de rhenanus (parte superior) - Zona superior de disparilis (Bultynck, 2003); Zona de ansatus - Zona de norrisi (Narkiewicz \& Bultynck, 2010); Zona de rhenanus - Zona superior de transitans (Liao \& Valenzuela-Ríos, 2008).

Género Polygnathus Hinde, 1879

Especie tipo Polygnathus dubius Miller, 1889 
Polygnathus decorosus Stauffer, 1938

(Figs. 8.1a-b, 8.2-8.3)

*1938 Polygnathus decorosus n. sp.; Stauffer, p. 438, Pl. 53, Figs. 1, 5, 6, 10, 11, 15, 16, 20 y 30.

1966 Polygnathus decorosa; Anderson, p. 411, Pl. 50, Figs. 6-8, 10, 11, 15, 19.

1983 Polygnathus decorosus; van de Boogaard, p. 6, Pl. 4, Figs. 5-8, 10.

1982 Polygnathus decorosus; Morzadec \& Weyant, p. 33, Pl. 2, Figs. 15-18.

1983 Polygnathus decorosus; Raven, Pl. 3, Figs. 7ab, 8a-b.

1985 Polygnathus decorosus; Klapper \& Lane, p. 935, Fig. 18.7.

1992 Polygnathus decorosus; Carls \& Gong, Pl. 4, Figs. 9-11.

1992 Polygnathus decorosus; Ji et al., Pl. IV, Figs. 19-20.

1993 Polygnathus decorosus; Ji \& Ziegler, p. 77, Pl. 40, Figs. 16-18.

1999 Polygnathus decorosus; Sanz-López et al., Pl. 1, Fig. 9.

1999 Polygnathus decorosus; Talent et al., P1. 6, Fig. 6. 2000 Polygnathus decorosus; Over \& Rhodes, p. 106, Figs. 4.12-4.13.

2001 Polygnathus decorosus; Liao et al., p. 24, Pl. 4, Fig. 11.

2007 Polygnathus decorosus; Gereke, Pl. 24, Fig. 3.

2007 Polygnathus decorosus; Over, Pl. 12, Figs. 13-14.

2008 Polygnathus decorosus; Ovnatanova \& Kononova, p. 1121, Pl. 17, Figs. 8-11.

2011 Polygnathus decorosus; Narkiewicz \& Bultynck, Pl. 5, Figs. 5-7; Pl. 9, Figs. 12, 17.

2014 Polygnathus decorosus; Rodríguez-Cañero \& Martín-Algarra, Fig. 10.7.

2015 Polygnathus decorosus; Mahboubí \& Gatovsky, Figs. 5C, D, J, K y L.

2015 Polygnathus decorosus; Soboleva \& Sobolev, Pl. 3, Figs. 17, 22 y 23.

2017 Polygnathus decorosus; Ovnatanova et al., p. 1120, Pl. 26, figs. 7-8; Pl. 41, Figs 10-11.

Número de elementos P1. 343 ejemplares.
Descripción. El elemento $\mathrm{P}_{1}$ se caracteriza por tener una plataforma sagitada, estrecha y ligeramente asimétrica. La lámina libre, algo más larga que la plataforma, presenta los dentículos de mayor altura en su parte ventral. Sus márgenes laterales están algo elevados y ornamentados con pequeñas costillas subperpendiculares a la carena. Presenta surcos adcarenales estrechos y someros. Su carena, que alcanza el extremo dorsal de la plataforma, está constituida por 4 o 5 altos nódulos fusionados y más anchos en la parte intermedia de la misma. Su pequeña cavidad basal se encuentra en el extremo ventral de la plataforma y tiene forma ovalada.

Observaciones. Véanse las observaciones de Polygnathus dubius sobre las diferencias con esta última especie.

Distribución bioestratigráfica. Zona de disparilis Zona de linguiformis (Ji \& Ziegler, 1993); según Liao \& Valenzuela-Ríos (2001) esta especie es exclusivamente Frasniense.

Polygnathus ovatinodosus Ziegler \& Klapper, 1976 (Figs. 8.4a-b)

*1976 Polygnathus ovatinodosus n. sp.; Ziegler et al., p. 124, Pl. 2, Figs. 1-9.

1980 Polygnathus ovatinodosus; Perri \& Spalletta, p. 306, Pl. 7, Figs. 4a-b.

1983 Polygnathus ovatinodosus; Raven, Pl. 4, Fig. 18.

1987 Polygnathus ovatinodosus; García-López, p. 95, P1. 12, Figs. 14-25.

1992 Polygnathus ovatinodosus; Bardashev, Pl.5, Figs. 39, $42-48$.

2001 Polygnathus ovatinodosus; Aboussalam \& Becker, Pl. 2 Fig. 1.

2002 Polygnathus ovatinodosus; García-López \& Bastida, Pl. 1, Fig. 23-24.

2002 Polygnathus ovatinodosus; García-López et al., Pl. 1, Figs. 23, 24.

2003 Polygnathus ovatinodosus; Aboussalam, p. 187, Pl. 15, Fig. 16; Pl. 16, Fig. 1.

2007 Polygnathus ovatinodosus; Aboussalam \& Becker, Fig. 7 I.

2008 Polygnathus ovatinodosus; Gholamalian \& Kebriaei, p.187, Pl. 2, Fig. 29.

2010 Polygnathus ovatinodosus; Narkiewicz \& Bultynck, Figs. 19. 9 y 19. 10. 
Número de elementos P1. 21 ejemplares.

Descripción. El elemento $\mathrm{P}_{1}$ tiene una plataforma ovalada, bastante plana y adornada por pequeños y numerosos nódulos que cubren toda su superficie. Presenta un pequeño rostrum en su parte ventral con surcos adcarenales poco profundos. La plataforma es un poco más larga que la lámina libre. Su orificio basal es redondeado y se encuentra situado bajo el rostrum.

Observaciones. El ejemplar figurado presenta un rostrum más reducido que el holotipo de Ziegler \& Klapper (1976), aunque encaja bien con los paratipos figurados.

Distribución bioestratigráfica. Zona media de varcus (Aboussalam \& Becker, 2001); Zona de hermanni - Zona MN2 (Aboussalam \& Becker, 2007); Gholamalian \& Kebriaei (2008), lo encuentran en la zona inferior-media de falsiovalis (MN1 - MN2).

Polygnathus dengleri Bischoff \& Ziegler, 1957

(Figs. 8.5a-b, 8.6)

*1957 Polygnathus dengleri n. sp.; Bischoff \& Ziegler, p. 87, P1. 15, Figs. 14, 15, 17-24; Pl. 16, Figs. 1-4.

1978 Polygnathus dengleri; Orchard, P1. 115, Figs. 20, 21, 24 .

1980 Polygnathus dengleri; Johnson et al., p. 102, Pl. 4, Figs. 24-28, 30.

1980 Polygnathus dengleri; Perri \& Spalletta, p. 305, P1. 7, Figs.1a-2b,

1992 Polygnathus dengleri; Bardashev, Pl. 8, figs. 25, 27-33; Pl. 10, Figs. 15, 16.

2001 Polygnathus dengleri; Liao et al., p. 26, Pl. 4, Figs. 7-9, 12.

2007 Polygnathus dengleri dengleri; Aboussalam \& Becker, Figs. 6 C-F.

2007 Polygnathus dengleri; Over, Pl. 10, Fig. 17.

2010 Polygnathus dengleri dengleri; Narkiewicz \& Bultynck, Figs. 16.1-16.2; Fig. 17.16-17.17.

2014 Mesotaxis dengleri; Rodríguez-Cañero \& MartínAlgarra, Fig. 9.1.

2017 Polygnathus dengleri dengleri; Ovnatanova et al., p. 1123, P1. 4, Fig. 9; Pl. 25, Fig.1.

Número de elementos P1. 111 ejemplares.

Descripción. El elemento $\mathrm{P}_{1}$ posee una plataforma ovalada y simétrica con el extremo dorsal aguzado y los márgenes laterales ligeramente flexionados hacia arriba. Está ornamentada con costillas no muy fuertes, relativamente espaciadas y dispuestas perpendicularmente a la carena, de la que están separadas por surcos adcarenales. La carena está formada por nódulos fusionados y bastante altos, que sobrepasan en altura a los márgenes laterales. La lámina libre es corta. El orificio basal es grande, alargado y simétrico y se sitúa un poco más ventralmente que el centro de la plataforma.

Observaciones. Johnson et al. (1980) indicaron que los especímenes de elementos $\mathrm{P}_{1}$ de Polygnathus dengleri de la Zona inferior de dengleri están caracterizados por una ornamentación grosera, con nódulos y costillas irregularmente repartidos, mientras que los de la Zona superior de dengleri e inferior de asymmetricus poseen una ornamentación más fina, con costillas transversales más débiles. Los aquí hallados pertenecen a este último tipo.

Distribución. Zona superior de disparilis - Zona de transitans (Ziegler \& Sandberg, 1990; Ovnatanova et al., 2017).

\section{Polygnathus dubius Hinde, 1879}

(Figs. 8.7a-b)

* 1879 Polygnathus dubius n. sp.; Hinde, p. 362, Pl. XVI, Figs. 15-18.

1971 Polygnathus dubius; Klapper \& Philip, p. 443, Fig. 12 P.

1980 Polygnathus dubius; Johnson et al., Pl. 4, Fig. 32.

1980 Polygnathus dubius; Perri \& Spalletta, p. 305, Pl. 7, Figs. 3a-b.

1982 Polygnathus dubius; Morzadec \& Weyant, p. 33, P1. 3, Figs. 9-11.

1983 Polygnathus dubius; Raven, Pl.3, Figs. 9a-b.

1985 Polygnathus dubius; Racki, Pl. 6, Figs. 5, 9.

1987 Polygnathus dubius; García-López, p. 88, Pl. 14, Figs. 4-16.

1992 Polygnathus dubius; Bardashev, P1.9, Figs. 6-8, 11-13, 15, 16, 23, 24.

1992 Polygnathus dubius; Carls \& Gong, Pl. 4, Figs. 4, 5.

1993 Polygnathus dubius; Ji \& Ziegler, p. 77, Pl. 40, Figs. 21, 23; P1. 41, Figs. 1-8.

2001 Polygnathus dubius; Liao et al., p. 26, Pl. 4, Figs. 14, 18, 19.

2007 Polygnathus dubius; Over, Pl. 10, Figs. 9-12. 
2008 Polygnathus dubius; Liao \& Valenzuela-Ríos, Fig. 5 A.

2011 Polygnathus dubius; Aboussalam \& Becker, p. 150, Fig. 9.16.

2012 Polygnathus dubius; Liao \& Valenzuela-Ríos, p. 832, Figs. 6 X, AB.

2013 Polygnathus dubius; Gholamalian et al., Pl. 2, Figs. 14, 16, 17.

2014 Polygnathus dubius; Rodríguez-Cañero \& MartínAlgarra, Fig. 10. 6.

2017 Polygnathus dubius; Ovnatanova et al., Pl. 2, Fig. 5; Pl. 4, Fig. 8; Pl. 6, Fig. 4; Pl. 7, Fig. 4; Pl. 9, Fig. 8; Pl. 10, Fig. 3; Pl. 25, Fig. 4.

Número de elementos P1. 120 ejemplares.

Descripción. El elemento $\mathrm{P}_{1}$ presenta una plataforma asimétrica con una amplia curvatura, un mayor desarrollo del lado rostral y una pequeña constricción a modo de rostrum en su parte ventral. Sobre los laterales de la plataforma desarrolla unas pequeñas costillas perpendiculares a la carena en la parte ventral y media, que se transforman en pequeños tubérculos en la parte dorsal. La carena está formada por nódulos alargados y algo fusionados y los surcos adcarenales terminan antes de llegar a su extremo dorsal. En su lámina libre se desarrollan dentículos comprimidos y su longitud es algo menor que la de la plataforma. El orificio basal se encuentra situado en el extremo ventral de la plataforma.

Observaciones. Polygnathus dubius difiere de $P$. decorosus por tener una plataforma más asimétrica y ancha, y por presentar una constricción en su parte ventral. Asimismo, su lámina libre es más corta.

Distribución bioestratigráfica. La exacta distribución de esta especie no está claramente establecida. Según Ziegler (1973) abarca desde la Zona superior de hermanni - cristatus hasta la Zona inferior de asymmetricus; para Ji \& Ziegler (1993) se extiende desde la Zona de varcus a la Zona de jamiae; y para Klapper \& Philip (1971) iría desde la Zona de hermanni - cristatus a la Zona de hassi.

Polygnathus linguiformis linguiformis Hinde, 1879 (Figs. 8.8a-b)

1879* Polygnathus linguiformis n. sp.; Hinde, p. 367, Pl. XVII, Fig. 15.

1967 Polygnathus linguiformis linguiformis; Adrichem Boogaert, p. 184, Pl. 2, Fig. 44; Pl. 3, Fig. 1.
1976 Polygnathus linguiformis linguiformis; GarcíaLópez, Pl. 1, Figs. 2A, 2B.

1976 Polygnathus linguiformis linguiformis gamma morphotype; Ziegler et al., p. 122, Pl. 4, Figs. 9, 13.

1977 Polygnathus linguiformis linguiformis; Weddige, Pl. 5, Figs. 80, 82.

1980 Polygnathus linguiformis linguiformis gamma morphotype; Bultynck \& Hollard, p. 43, P1.VII, Fig. 1.

1985 Polygnathus linguiformis linguiformis; Olivieri, p. 302, Pl. 2, Figs. 17-21.

1987 Polygnathus linguiformis linguiformis; GarcíaLópez, p. 93, Pl. 13, Figs. 1-10.

1989 Polygnathus linguiformis linguiformis; Mawson \& Talent, Pl. 5, Figs. 1-3.

1990 Polygnathus linguiformis linguiformis gamma morphotype; Uyeno, p. 84, P. 18, Figs. 5-7, 9.

1992 Polygnathus linguiformis linguiformis gamma morphotype; Bardashev, Pl. 3, Figs. 12, 18, 19, 25.

1992 Polygnathus linguiformis linguiformis; Ji et al., P1. IV, Fig. 24.

1999 Polygnathus linguiformis linguiformis gamma morphotype; Lazreq, Pl. 1, Fig. 20-21.

2001 Polygnathus linguiformis linguiformis; Liao et al., p. 29, Pl. 2, Figs. 5-13, 15-18.

2001 Polygnathus linguiformis linguiformis gamma morphotype; Ovnatanova \& Kononova, p. 43, Pl. 1, Fig. 2.

2003 Polygnathus linguiformis linguiformis; Aboussalam, p.181, Pl. 16, Fig. P; Pl. 17, Figs. 1-6.

2008 Polygnathus linguiformis linguiformis; Liao \& Valenzuela-Ríos, Figs. 3C-D.

2012 Polygnathus linguiformis linguiformis; Matyja, Figs. 17L-N.

2013 Polygnathus linguiformis linguiformis; Gholamalian et al., Pl. 3, Fig. 19.

2013 Polygnathus linguiformis linguiformis; Gouwy, p. 265, Pl. 3, Figs. 3-5.

2015 Linguipolygnathus linguiformis linguiformis (gamma morphotype); Mahboubi et al., Fig. 5C.

Número de elementos P1. 6 ejemplares.

Descripción. El elemento $P_{1}$ posee una gran plataforma rectangular que termina con un brusco giro para formar la lengua; el margen rostral describe un fuerte ángulo al comienzo de la lengua mientras que el caudal se curva 
suave y uniformemente. En su parte ventral presenta los márgenes fuertemente elevados con grandes y profundos surcos adcarenales que se hacen menos marcados hacia la parte central y terminan al comienzo de la lengua. Ésta representa casi una tercera parte de la plataforma y está ornamentada con costillas transversales. La carena se interrumpe al comienzo de la lengua y está formada por dentículos altos en la parte ventral y por otros más bajos $\mathrm{y}$ fusionados en la dorsal.

Observaciones. El ejemplar fotografiado carece de la lámina libre pero el resto de sus características permite asignarlo a esta especie con suficiente seguridad.

Distribución bioestratigráfica. Parte inferior del Devónico Medio - Zona de asymmetricus (Ziegler, 1977); según Gouwy (2013) comienza en la Zona de timorensis; Devónico Medio bajo (Eifeliense superior alto) hasta el Devónico Superior (Frasniense; Zona asymmetricus, Liao et al., 2001).

\section{Polygnathus cf. timorensis Klapper, Philip \& Jackson,} 1970

(Fig. 8.9)

1970 Polygnathus timorensis n. sp.; Klapper et al., p. 655, Pl. 1, Figs. 1-3 y 7-10.

1980 Polygnathus timorensis; Johnson et al., Pl. 3, Fig. 38.

1983 Polygnathus timorensis; Raven, Pl. 3, Fig. 3.

1987 Polygnathus timorensis; García-López, p. 98, Pl. 10, Figs. 20-29.

1987 Polygnathus timorensis; Bultynck, p. 162, Pl. 7, Figs. 9-10.

1989 Polygnathus timorensis; Mawson \& Talent, PL. 4, Figs. 7-12.

1992 Polygnathus timorensis; Bardashev, Pl. 5, Figs. 15-17, 24-26, 28-30.

1992 Polygnathus timorensis; Carls \& Gong, Pl. 1, Fig. 20.

1992 Polygnathus timorensis; Ji et al., Pl. IV, Figs. 1-4.

1999 Polygnathus timorensis; Lazreq, p. 76, P1. 2, Figs. 5-8.

1999 Polygnathus timorensis; Talent et al., Pl. 6, Figs. $1-2$.

2001 Polygnathus timorensis; Liao et al., p. 38, Pl. 4, Figs. 4-6.

2003 Polygnathus timorensis; Aboussalam, p. 188, P1. 15, Figs. 9-13.
2008 Polygnathus timorensis; Liao \& Valenzuela-Ríos, p. 9, Fig. 3E-F.

2013 Polygnathus timorensis; Gouwy, p. 266, Pl. 2, Figs. 11-13.

2013 Polygnathus timorensis; Liao \& Valenzuela-Ríos, p. 361, Figs. 8B.

Número de elementos P1. 4 ejemplares.

Descripción. El elemento $\mathrm{P}_{1}$ presenta una plataforma alargada y estrecha con surcos adcarenales profundos. $\mathrm{Su}$ parte ventral es asimétrica ya que los extremos cóncavos de sus márgenes ventrales tienen mayor desarrollo en el lado rostral que en el caudal, uniéndose a la lámina libre a diferente altura. Los puntos de geniculación también se hallan ligeramente desplazados. La plataforma está ornamentada con nódulos que se disponen sobre los bordes laterales de la misma a continuación de los puntos de geniculación.

Observaciones. El ejemplar fotografiado se encuentra fragmentado y conserva la plataforma solo parcialmente, razón por la cual se asigna a esta especie con ciertas dudas. No obstante, las partes que se conservan encajan bien con las características de esta especie.

Distribución bioestratigráfica. Zona de varcus - Zona de disparilis (Aboussalam, 2003).

\section{DISCUSIÓN}

\subsection{Correlación con otros sectores del Complejo Maláguide}

Estudios previos en la UMS de la ZCV han identificado niveles carbonatados que han podido ser datados mediante conodontos en otros afloramientos de una sucesión pizarrosa de características semejantes a la denominada en este trabajo sucesión pre-Falcoña. En el afloramiento de las Mimbres Navas-Parejo et al. (2011) encontraron Criteriognathus steinhornensis en unas calizas tableadas con dacrioconáridos e intercalaciones pizarrosas de aproximadamente un metro de potencia, lo que les permitió datar el Emsiense (Devónico Inferior). En La Solana Navas-Parejo et al. (2015) dataron otro nivel calcáreo como Fameniense (Devónico Superior), concretamente de las zonas superior y más superior de crepida, mediante Palmatolepis angularis, $P a$. crepida, $P a$. quadrantinodosalobata y $P$ a regularis, además de otros niveles del Viseense, pertenecientes al miembro superior de la Fm. Falcoña. En las proximidades del Cortijo de la 
Ermita, unos 300 metros NW del afloramiento estudiado, un nivel semejante ha proporcionado también abundantes Pseudooneotodus beckmanni y dacrioconáridos (datos propios inéditos), lo que podría indicar que se trata de un Devónico Inferior equivalente al de las Mimbres. Sin embargo, no había sido mencionada anteriormente la existencia del Frasniense.

En otros afloramientos del Complejo Maláguide sí se han datado niveles de calizas del Frasniense mediante conodontos. Kockel \& Stoppel (1962) estudiaron, en la región de Almogía (Málaga), varias muestras que dataron como Adorfense-Nehdenense (Frasniense). En la misma área, Rodríguez-Cañero (1993) identificó las biozonas de transitans (Frasniense inferior), rhenana y linguiformis (Frasniense superior). Rodríguez-Cañero (1993) indicó la presencia de la zona de transitans en cantos del conglomerado del Miembro Retamares en Fuengirola. Herbig (1985) citó la existencia de la zona superior de gigas en el arroyo de la Cruz (Marbella). En fin, en un conglomerado depositado en relación con los eventos del límite Frasniense-Fameniense en el afloramiento de la Falcoña Alta, al NW de la ciudad de Málaga, RodríguezCañero \& Martín-Algarra (2014) identificaron clastos con asociaciones de conodontos del Frasniense de las zonas de superior de falsiovalis-inferior de transitans, transitans, superior de punctata-inferior de hassi, y superior de rhenana. En ellas identificaron seis biofacies que revelan una variada procedencia desde ambientes de plataforma a pelágicos profundos.

La fauna Frasniense de las zonas MN1 y MN2 hallada en el nivel 17A-67 de la ZCV es, sin embargo, más antigua que las presentes en los restantes afloramientos del Frasniense conocidos en el Complejo Maláguide y es, por tanto, el Frasniense más antiguo hallado en el mismo. La biofacies de polygnáthidos que contiene sí está presente también en el afloramiento de la Falcoña Alta, pero en las zonas de transitans y de punctata-hassi (Rodríguez-Cañero \& Martín-Algarra, 2014), así como en el Givetiense superior (Zona de disparilis) del área de Almogía (Rodríguez-Cañero, 1993; Rodríguez-Cañero \& Martín-Algarra, 2014).

La datación realizada en el nivel aquí estudiado y la presencia, previamente reconocida en la $\mathrm{ZCV}$, de otros horizontes carbonatados con conodontos del Devónico, permite correlacionar la sucesión pre-Falcoña de la ZCV con otras pertenecientes al Complejo Maláguide, dentro del Grupo Piar, particularmente con la Formación Santi Petri. Esta última formación no ha podido ser datada hasta la fecha, a pesar de numerosos intentos realizados en distintos afloramientos del Complejo Maláguide (por ejemplo, Kockel, 1959; Kockel \& Stoppel, 1962; Herbig, 1985; Rodríguez-Cañero, 1993) ni tampoco en el sector estudiado de la ZCV. Sin embargo, tradicionalmente se le ha atribuido una edad Devónico por su posición estratigráfica subyacente a la Fm. Falcoña, de edad Tournaisiense-
Viseense (Rodríguez-Cañero \& Guerra-Merchán, 1996, O'Dogherty et al., 2000), que es equivalente a la que ocupa la sucesión pre-Falcoña de la UMS de la ZCV con respecto a dicha formación.

\subsection{Implicaciones paleogeográficas}

El depósito de la sucesión pre-Falcoña aconteció en ambientes marinos abiertos dominantemente terrígenos y de baja energía, a los que esporádicamente llegaban aportes detríticos algo más gruesos. Estos sedimentos, que forman niveles aislados dentro de una sucesión dominantemente pizarrosa, se depositaron bajo condiciones de mayor energía, que generaban estructuras de corrientes, huellas de muro, granoclasificación y laminación cruzada. Estos niveles son de tres tipos: i) areníticos finos, cuarcíticos y ligeramente micáceos, ii) volcanoclásticos (localmente asociados a lavas y/o rocas subvolcánicas) y iii) carbonatados calciclásticos, por lo general de grano fino a muy fino.

En este contexto sedimentario, la relación original de la sucesión pre-Falcoña con las facies de "calizas alabeadas" de la Fm. Santi Petri, ampliamente presentes en la UMI, se interpreta como un cambio lateral de facies. Los horizontes carbonatados de la sucesión preFalcoña son de mayor granulometría y menos continuos, potentes o abundantes que los que aparecen en la Fm. Santi Petri. Por tanto, las características sedimentológicas y las biofacies de la sucesión pre-Falcoña indican que su depósito aconteció en ambientes más proximales que los de la Fm. Santi Petri, cuyos sedimentos clásticos profundos son propios de una zona más distal dentro del mismo margen continental.

\section{CONCLUSIONES}

1) En la Unidad Maláguide Superior de la Zona de Cogollos Vega (provincia de Granada) se ha reconocido, por primera vez, un nivel fosilífero del Frasniense inferior que, además, es el más bajo de este piso encontrado hasta la fecha en el Complejo Maláguide y que se correlaciona con las zonas MN1 y MN2 de la Montaña Negra.

2) La biofacies de polygnáthidos indica que la población de conodontos estudiada procedía de ambientes marinos abiertos moderadamente profundos o incluso relativamente someros, situados en una zona relativamente proximal de un margen continental.

3) El nivel datado se intercala en una sucesión de pelitas con niveles areníticos siliciclásticos y volcanoclásticos que subyace a la Fm. Falcoña. Por ello, dicha sucesión ha sido informalmente denominada sucesión pre-Falcoña. Esta sucesión también intercala otros niveles carbonatados, algunos de los cuales han proporcionado fauna de 
conodontos del Devónico, más antigua (Emsiense) y más moderna (Fameniense).

4) La sucesión pre-Falcoña no se encuentra en relación de afloramiento con las facies de calizas alabeadas de la Fm. Santi Petri, la más característica y ampliamente representada del Devónico Maláguide, que ha sido identificada en una unidad tectónica independiente. Sin embargo, por su edad y posición estratigráfica con respecto a la Fm. Falcoña, ambas sucesiones, pre-Falcoña y Santi Petri, deben haberse depositado contemporáneamente: la primera en ambientes más proximales y menos profundos y la segunda en ambientes netamente turbidíticos, distales y más profundos.

\section{AGRADECIMIENTOS}

Agradecemos las cuidadosas revisiones de las Dras. Pilar Navas-Parejo, Jau Chyn Liao, y al editor de la revista Dr. Carlos Martínez Pérez, que nos han ayudado a mejorar el presente trabajo. Este estudio recoge y amplía los resultados obtenidos por la primera firmante durante la realización de sus Trabajos de Fin de Grado (TFG) y de Fin de Máster (TFM) bajo la supervisión de los otros dos firmantes. La investigación ha sido financiada con fondos del proyecto CGL2016-75679P (MINECO/FEDER) y del grupo RNM-208 (Junta de Andalucía). Agradecemos a las Dras. Isabel Sánchez-Almazo y Rocío Márquez Crespo (C.I.C.-UGR) la ayuda prestada durante la realización del estudio mediante SEM.

\section{BIBLIOGRAFÍA}

Aboussalam, Z.S. 2003. Das “Taghanic-Event” im höheren Mittel-Devon von West Europa und Marokko. PhD Thesis, Münstersche Forschungen zur Geologie und Palaeontologie, 97, 1-332.

Aboussalam, Z.S. \& Becker, R. 2001. Prospects for an upper Givetian substage. Fossil Record, 4, 83-99; doi:10.1002/ mmng.20010040107.

Aboussalam, Z.S. \& Becker, T. 2007. New upper Givetian to basal Frasnian conodont faunas from the Tafilalt (AntiAtlas, Southern Morocco). Geological Quarterly, 51, 345-374.

Aboussalam, Z.S. \& Becker, T. 2011. The global Taghanic Biocrisis (Givetian) in the eastern Anti-Atlas, Morocco. Palaeogeography, Palaeoclimatology, Palaeoecology, 304, 136-164; doi: 10.1016/j.palaeo.2010.10.015.

Adrichem Boogaert, H.A. Van. 1967. Devonian and Lower Carboniferous conodonts of the Cantabrian Mountains (Spain) and their stratigraphic application. Leidse Geologische Mededelinge, 39, 129-192.
Anderson, M.A. 1966. Upper Devonian conodonts and the Devonian-Mississippian boundary of North-Central Iowa. Journal of Paleontology, 40, 395-415.

Bahrami, A., Zamani, F., Corradini, C., Yazdi, M. \& Ameri, H. 2014. Late Devonian (Frasnian) conodonts from the Bahram Formation in the Sare-Ashk Section, Kerman Province, Central-East Iran Microplate. Bollettino della Società Paleontologica Italiana, 53, 179-188; doi:10.4435/BSPI.2014.15.

Bardashev, I.A. 1992. Conodont stratigraphy of middle Asia middle Devonian. Courier Forschungsinstitut Senckenberg, 154, 31-83.

Bateson, W. 1886. The ancestry of the Chordata. Quarterly Journal of Microscopical Sciences, 26, 535571.

Bischoff, G. \& Ziegler, W. 1957. Die Conodontenchronologie des Mitteldevons und des tiefsten Oberdevons. Abhandlungen des Hessischen Landesamtes für Bodenforschung, 22, 1-136.

Blumenthal, M. 1927. Versuch einer tectonischen Gliederung der Betischen Cordilleren von Central und Südwest Andalusien. Eclogae Geologicae Helvetiae, 20, 487-592.

Blumenthal, M. 1949. Estudio geológico de las cadenas costeras al oeste de Málaga entre el río Guadalhorce y el río Verde. Boletín del Instituto Geológico y Minero de España, 62, 1-191.

Blumenthal, M. \& Fallot, P. 1935. Observations géologiques sur la Sierra Arana, entre Grenade et Guadix. Memorias de la Sociedad Española de Historia Natural, 17, 5-74.

Boersma, K.Th. 1973. Devonian and Lower Carboniferous conodont biostratigraphy, Spanish Central Pyrenées. Leidse Geologische Mededelinge, 49, 303-337.

Branson, E.B. 1938. Stratigraphy and paleontology of the Lower Mississippian of Missouri. Part I. University of Missouri Studies, 13, 1-20.

Branson, E.B. \& Mehl, M.G. 1934. Conodonts from the Grassy Creek shale of Missouri. University of Missouri Studies, VIII, 172-25.

Branson, E.B. \& Mehl, M.G. 1938. The conodonts genus Icriodus and its stratigraphic distribution. Journal of Paleontology, 12, 156-166.

Bultynck, P. 1987. Pelagic and neritic conodont successions from the Givetian of pre-Sahara Morocco and the Ardennes. Bulletin de l'Institut Royal des Sciences Naturelles de Belgique, Sciences de la Terre, 57, 149181.

Bultynck, P. 2003. Devonian icriodontidae: Biostratigraphy, classification and remarks on paleoecology and dispersal. Revista Española de Micropaleontología, 35, 295-314.

Bultynck, P. \& Hollard, H. 1980. Distribution comparée de Conodonts et Goniatiles dévoniens des plaines du Dra, du Máder et du Tafilalt (Maroc). Aardkundige Mededelingen, 1, 7-76.

Çapkinoglu, S. \& Gedik, I. 2000. Late Devonian conodont fauna of the Gümüsali Formation, the Eastern Taurides, Turkey. Turkish Journal of Earth Sciences, 9, 69-89.

Carls, P. \& Gong, D. 1992. Devonian and early Carboniferous conodonts from Shidian (Western Yunnan, China). Courier Forschungsinstitut Senckenberg, 154, 179-221. 
Carls, P. \& Valenzuela-Ríos, J.I. 2002. Early Emsian Conodonts and associated shelly faunas of the Mariposas Fm (Iberian Chains, Aragon, Spain). In: Palaeozoic Conodonts from Northern Spain (eds. García-López, S. \& Bastida, F.). Publicaciones del Instituto Geológico y Minero de España. Cuadernos del Museo Geominero, 1, 299-314.

Draganits, E., Mawson, R., Talent, J. \& Krystyn, L. 2002. Lithostratigraphy, conodont biostratigraphy and depositional enviroment of the Middle Devonian (Givetian) to Early Carboniferous (Tournaisian) Lipak Formation in the Pin Valley of Spiti (NW India). Rivista Italiana di Paleontologia e Stratigrafia, 108, 7-35; doi: 10.13130/2039-4942/5450.

Durand-Delga, M.M. 1968. Coup d'oeil sur les unités Malaguides des Cordillères Bétiques (Espagne). Comptes Rendus de l'Académie des Sciences de Paris, 266, 190-193.

Dzik, J. 1976. Remarks on the evolution of Ordovician conodonts. Acta Palaeontologica Polonica, 21, 395-455.

Epstein, A.G., Epstein, J.B. \& Harris, L.D. 1977. Conodont Color Alteration-an Index to Organic Metamorphism. $U$. S. Geological Survey professional paper, 995, 1-27; doi: https://doi.org/10.3133/pp995.

García-Dueñas, V. \& Navarro-Vilá, F. 1976. Alpujarrides, Malaguides, et autres unités allochtones au Nord de la Sierra Nevada (Cordillères Bétiques, Andalousie). Bulletin de la Société Géologique de France, 18, 641-648.

García-López, S. 1976. La caliza de Candas en la zona de Luanco (Asturias) y su contenido en conodontos. Trabajos de Geología, 8, 173-185.

García-López, S. 1984. Terminología morfológica de los conodontos compuestos y de plataforma. Revista de Biología de la Universidad de Oviedo, 2, 95-105.

García-López, S. 1987. Los Conodontos y su Aplicación al Estudio de las Divisiones Cronoestratigráficas Mayores del Devónico Asturleonés (España). Publicaciones especiales del Boletín Geológico y Minero, pp 112.

García-López, S \& Bastida, F. (eds.) 2002. Palaeozoic Conodonts from Northern Spain. Publicaciones del Instituto Geológico y Minero de España. Cuadernos del Museo Geominero.

García-López, S. \& Sanz-López, J. 2002. Devonian to Lower Carboniferous conodont biostratigraphy of the Bernesga Valley section (Cantabrian Zone, NW Spain). In: Palaeozoic Conodonts from Northern Spain (eds. García-López, S. \& Bastida, F.). Publicaciones del Instituto Geológico y Minero de España. Cuadernos del Museo Geominero, 1, 163-205.

García-López, S. \& Sanz-López, J. (with contribution by Sarmiento, G.N.). 2002. The Palaeozoic succession and conodont biostratigraphy of the section between Cape Peñas and Cape Torres (Cantabrian coast, NW Spain). In: Palaeozoic Conodonts from Northern Spain (eds. GarcíaLópez, S. \& Bastida, F.). Cuadernos del Museo Geominero, 1, 125-161.

Gereke, M. 2007. Die oberdevonische Kellwasser-Krise in der Beckenfazies von Rhenoherzynikum und Saxothuringikum (spätes Frasnium/frühestes Famennium, Deutschland). Kölner Forum für Geologie und Paläontologie, 17, 1-228.
Gholamalian, H. \& Kebriaei, M. 2008. Late Devonian conodonts from the Hojedk section, Kerman Province, southeastern Iran. Rivista Italiana di Paleontologia e Stratigrafia, 114, 179-189; doi: 10.13130/2039-4942/5898.

Gholamalian, H., Hairapetian, V., Barfehei, N., Mangelian, S. \& Faridi, P. 2013. Givetian-Frasnian boundary conodonts from Kerman province, central Iran. Rivista Italiana di Paleontologia e Stratigrafia, 119, 133-146; doi: 10.13130/2039-4942/6028.

Gouwy, S. 2013. New data on Middle Devonian conodonts from SW-Sardinia: the Su Nuargi II revisited. Rivista Italiana di Paleontologia e Stratigrafia, 119, 257-273; doi: 10.13130/2039-4942/6039.

Gouwy, S., Liao, J.C. \& Valenzuela-Ríos, J.I. 2013. Eifelian (Middle Devonian) to Lower Frasnian (Upper Devonian) conodont biostratigraphy in the Villech section (Spanish Central Pyrenees). Bulletin of Geosciences, 88, 315-338; doi: 10.3140/bull.geosci.1341.

Hass, W.H. 1959. Conodonts of the Barnett Formation of Texas. U.S. Geological Survey professional paper, 243, 69-94; doi: https://doi.org/10.3133/pp243F.

Herbig, H.G. 1983. El Carbonífero de las Cordilleras Béticas. In: Carbonífero y Pérmico de España (ed. Martínez Díaz, C.). X Congreso Internacional de Estratigrafía y Geología del Carbonífero, Madrid, 343-356.

Herbig, H.G. 1984. Rekonstruktion eines nicht mehr existenten Sedimentationsraumes-Die Kalkgerölle im Karbon-Flysch der Malagiden (Betische Kordillere, Südspanien). Facies, 11, 1-108; doi: 10.1007/BF02536907.

Herbig, H.G. 1985. An Upper Devonian Limestone Slide Block near Marbella (Betic Cordillera, Southern Spain) and the Palaeogeographic Relations between Malaguides and Menorca. Acta Geologica Hispanica, 20, 155-178.

Hinde, G.J. 1879. On conodonts from the Chazy and Cincinnati Group of the Cambro-Silurian, and from the Hamilton and Genessee-Shale divisions of the Devonian in Canada and the United States. Quarterly Journal of the Geological Society of London, 35, 351-369; doi: 10.1144/ GSL.JGS.1879.035.01-04.23.

Jeppsson, L., Anehus, R. \& Fredholm, D. 1999. The optimal acetate buffered acetic acid technique for extracting phosphatic fossils. Journal of Paleontology, 73, 964-972.

Ji, Q. \& Zeigler, W. 1993. The Lali Section: An excellent Reference section for Upper Devonian in South China. Courier Forschungsinstitut Senckenberg, 188, 99-101.

Ji, Q., Ziegler, W. \& Dong, X. 1992. Middle and Late Devonian conodonts from the Licum section Yongfu, Guangxi, south China. Courier Forschungsinstitut Senckenberg, 154, 85-105.

Johnson, J.G., Klapper, G. \& Trojan, W.R. 1980. Brachiopod and Conodont successions in the Devonian of the northern Antelope Range, central Nevada. Geologica et Palaeontologica, 14, 77-116.

Khalymbadzha, V.G. \& Chernysheva, N.G. 1970. Conodonts of the genus Ancyrodella from the Devonian beds of the Volga-Kama Region and their stratigraphic significance. In: Biostratigrafiya i Paleontologiya Paleozoiskikh Otlozhenii Vostoka Russkoi Platformy i Zapadnogo 
Priural'ya [Biostratigraphy and Paleontology of the Paleozoic Beds of the Eastern Russian Platform and Western Fore-Urals]. Kazan Gos. University, 1, 81-103.

Klapper, G. 1989. The Montagne Noire Frasnian (Upper Devonian) conodont succession. In: Devonian of the World (eds. McMillan, N.J., Embry, A. \& Glass, D.J.). Canadian Society of Petroleum Geologist Memoir, 14, 449-468.

Klapper, G. 2000. Species of Spathognathodontidae and Polygnathidae (Conodonta) in the recognition of Upper Devonian stage boundaries. Courier Forschungsinstitut Senckenberg, 200, 153-159.

Klapper, G. \& Becker, R.T. 1998. Comparison of Frasnian (Upper Devonian) Conodont Zonations. In: ECOS VII, Seventh International Conodont Symposium (ed. Bagnoli, G.). Abstracts, 53-54.

Klapper, G. \& Becker, R.T. 1999. Comparison of Frasnian (Upper Devonian) Conodont zonations. Bollettino della Società Paleontologica Italiana, 37, 339-348.

Klapper, G. \& Johnson, J.G. 1990. Revisions of Middle Devonian conodont zones. In: Lower and Middle Devonian brachiopod-dominated communities of Nevada, and their position in a biofacies-province-realm model. Journal of Paleontology, 64, 934-941.

Klapper, G. \& Kirchgasser, W. 2016. Frasnian Late Devonian conodont biostratigraphy in New York: Graphic correlation and taxonomy. Journal of Paleontology, 90, 525-554; doi: 10.1017/jpa.2015.70.

Klapper, G. \& Lane, R.H. 1985. Upper Devonian (Frasnian) conodont of the Polygnathus biofacies, N.W.T., Canada. Journal of Paleontology, 59, 904-951.

Klapper, G. \& Philip, G.M. 1971. Devonian conodont apparatuses and their vicarious skeletal elements. Lethaia, 4, 429-452; doi: 10.1111/j.1502-3931.1971.tb01865.x.

Klapper, G., Philip, G.M. \& Jackson, J.H. 1970. Revision of the Polygnathus varcus group (Conodonta, Middle Devonian). Neues Jahrbuch für Geologie und Paläontologie, Monatshefte, 11, 650-667.

Kockel, F. 1959. Conodontos del Paleozoico de Málaga. Notas y Comunicaciones del Instituto Geológico y Minero de España, 53, 149-164.

Kockel, F. \& Stoppel, D. 1962. Nuevos hallazgos de conodontos y algunos cortes en el Paleozoico de Málaga (Sur de España). Notas y Comunicaciones del Instituto Geológico y Minero de España, 65, 133-170.

Kralick, J.A. 1994. The conodont genus Ancyrodella in the Middle Genesee Formation (Lower Upper Devonian, Frasnian), western New York, Journal of Paleontology, 68, 1384-1395; doi: 10.1017/S0022336000034351.

Lazreq, N. 1999. Biostratigraphie des conodontes du Givétien au Famennien du Maroc central. Biofaciès et événement Kellwasser. Courier Forschungsinstitut Senckenberg, 214, 1-111.

Liao, J.C. \& Valenzuela-Ríos, J.I. 2008. Givetian and Early Frasnian conodonts from the Compte section (MiddleUpper Devonian, Spanish Central Pyrenees). Geological Quarterly, 52, 1-118.

Liao J.C. \& Valenzuela-Ríos J.I. 2012. Upper Givetian and Frasnian (Middle and Upper Devonian) conodonts from
Ampriu (Aragonian Pyrenees, Spain): Global correlations and palaegeographic relations. Palaeontology, 55, 819842; doi: 10.1111/j.1475-4983.2012.01150.x.

Liao, J.C. \& Valenzuela-Ríos, J.I. 2013. The Middle and Upper Devonian conodont sequence from La Guardia D'Ares sections (Spanish Central Pyrenees), Bulletin of Geosciences, 88, 339-368; doi: 10.3140/bull.geosci.134.

Liao J.C., Valenzuela-Ríos J.I. \& Rodríguez, S. 2001. Descripción de los conodontos del Givetiense y Frasniense inferior (Devónico) de Renanué (Pirineos Aragoneses). Coloquios de Paleontología, 52, 13-45.

Mahboubi, A. \& Gatovsky, Y. 2015. Late Devonian conodonts and event stratigraphy in northwestern Algerian Sahara. Journal of African Earth Sciences, 101, 322-33; doi: 10.1016/j.jafrearsci.2014.10.004.

Mahboubi, A., Feist, R., Conée, J.J., Mehadji, A.O. \& Girad, C. 2015. Frasnian (Late Devonian) conodonts and environment at the northern margin of the Algerian Sahara platform: The Ben Zireg section. Geological Magazine, 152, 844-857; doi: 10.1017/S0016756814000715.

Martín-Algarra, A. 1987. Evolución geológica alpina del contacto entre las Zonas Internas y las Zonas Externas de la Cordillera Bética. $\mathrm{PhD}$ Thesis. Universidad de Granada, España. 1171 pp.

Martín-Algarra, A., Rodríguez-Cañero, R., O’Dogherty, L., Sánchez-Navas, A. \& Ruiz-Cruz, M.D. 2004. Complejo Maláguide. Estratigrafía. Paleozoico y más antiguo? (Grupo Piar). In: Geología de España (ed. Vera, J.A.). 401-404. Sociedad Geológica de España y Instituto Geológico y Minero de España, Madrid. 401-404.

Martín-Algarra, A., Mazzoli, S., Perrone, V., RodríguezCañero, R. \& Navas-Parejo, P. 2009a. Variscan tectonics in the Malaguide Complex (Betic Cordillera, Southern Spain): stratigraphic and structural Alpine vs. pre-Alpine constraints from the Ardales area (Province of Malaga). I: Stratigraphy. Journal of Geology, 117, 241-262; doi: 10.1086/597364.

Martín-Algarra, A., Mazzoli, S., Perrone, V. \& RodríguezCañero, R. 2009b. Variscan tectonics in the Malaguide Complex (Betic Cordillera, Southern Spain): stratigraphic and structural Alpine vs. pre-Alpine constraints from the Ardales area (Province of Malaga). II: Structure. Journal of Geology, 117, 263-284; doi: 10.1086/597365.

Matyja, H., 2012. Wyniki badań litologicznych, stratygraficznych i 1215 sedymentologicznych. Dewon. Stratygrafia i uwagi o wykształceniu facjalnym serii węglanowych żywetu i?franu. In: Profile Gtebokich Otworów Wiertniczych Państwowego Instytutu Geologicznego, Tuchola IG 1 (ed. Matyja H.). Seszyt, 135, 62-73. (En polaco). [Resultados de pruebas litológicas, estratigráficas y sedimentológicas. Devónico. Estratigrafía y notas sobre la formación de series de facies carbonatadas. In: Testigos de Sondeos Profundos, Servicio Geológico de Polonia, Tuchola IG 1 (ed. Matyja, H.)].

Mawson, R. \& Talent, J.A. 1989. Late Emsian-Givetian stratigraphy and conodont biofacies-carbonates slope and offshore shoal to sheltered lagoon and nearshore carbonate ramp - Broken River, North Queensland Australia. Courier Forschungsinstitut Senckenberg, 117, 205-259. 
Michelau, P. 1942. Das Paläozoikum der Betischen Ketten NW von Malaga. PhD Thesis. Universität Berlin, $26 \mathrm{p}$. (inédita).

Miller, C.G. 2007. Growth in early species of the conodont Ancyrodella and implications for correlation of the Middle-Upper Devonian boundary. Geological Quarterly, 51, 443-452.

Miller, S.A. 1889. North American Geology and Paleontology for the Use of Amateurs, Students and Scientists. Cincinnati, Ohio. West. Method. Book Conc.

Mon, R. 1971. Estudio geológico del extremo occidental de los Montes de Málaga y de la Sierra de Cártama (Prov. De Málaga). Boletin Geológico y Minero, 82-83, 132-141.

Morzadec, P. \& Weyant, M. 1982. Lithologie et Conodontes, de l'Emsien au Famennien, dans la rade de Brest Massif Armoricain. Geologica et Palaeontologica, 15, 27- 46.

Mouravieff, A.N. 1982. Conodont stratigraphic scheme of the Frasnian of the Ardennes. In: Papers on the FrasnianGivetian Boundary: Brussels, Geological Survey of Belgium and Subcommission on Devonian Stratigraphy, International Union of Geological Sciences (ed. Bigey, F. et al.). 101-118.

Müller, K.J. \& Müller, E.M. 1957. Early Upper Devonian (Independence) conodonts from Iowa, Part 1. Journal of Paleontology, 31, 1069-1108.

Narkiewicz, K. \& Bultynck, P. 2010. The upper Givetian (middle Devonian) subterminus conodont zone in North America, Europe and North Africa. Journal of Paleontology, 8, 588-625; doi: 0022-3360/10/0084$0588 \$ 03.00$.

Narkiewicz, K. \& Bultynck, P. 2011. Biostratygrafia konodontowa Dewonu Gornego Lubelszczyzny. Prace Państwowego Instytutu Geologicznego, 196, 193-254. (En polaco). [Upper Devonian Conodont biostratigraphy of the Lublin region].

Navarro-Vilà, F. \& García-Dueñas, V. 1979. Mapa y Memoria Explicativa de la Hoja 1010 (La Peza) del Mapa Geológico de España, a Escala 1:50.000 (2 ${ }^{a}$ Serie). IGME Madrid, 83 pp.

Navas-Parejo, P. 2012. Paleozoic stratigraphy and palaeogeography of the Malaguide Complex (Betic Cordillera) and other western Mediterranean related domains (Calabria-Peloritani Terrane). Ph.D. Thesis. Universidad de Granada. Departamento de Estratigrafía y Paleontología. 217 pp.

Navas-Parejo, P., Martín-Algarra, A. \& Martínez-Pérez, C. 2011. Primeros datos sobre la presencia de conodontos del Emsiense (Devónico Inferior) en el Complejo Maláguide de la provincia de Granada. Paleontologia i Evolució, Memòria especial, 5, 425-429.

Navas-Parejo, P., Rodríguez-Cañero, R. \& Martín-Algarra, A. 2015. New conodont data from a Devonian-Carboniferous succession in the central sector of the Betic Cordillera (SE Spain). Spanish Journal of Palaeontology, 30, 133-146.

O’Dogherty, L., Rodríguez-Cañero, R., Gursky, H.J., MartínAlgarra, A. \& Caridroit, M. 2000. New data on Lower Carboniferous stratigraphy and palaeogeography of the Malaguide Complex (Betic Cordillera, Southern Spain).
Comptes Rendus de l'Academie des Sciences Paris, 331, 533-541.

Olivieri, R. 1985. Middle and Late Devonian conodonts from Southwestern Sardinia. Bollettino della Società Paleontologica Italiana, 23, 269-310.

Orchard, M.J. 1978. The conodont biostratigraphy of Devonian Phymouth Limestone, south Devon. Palaentology, 21, 907-955.

Orueta, D. 1917. Estudio petrográfico de la Serranía de Ronda. Memorias del Instituto Geológico y Minero de España, $41,45-71$.

Over, D.J. 2007. Conodont biostratigraphy of the Chattanooga shale, Middle and Upper Devonian, Southern Appalachian basin Eastern United States. Journal of Paleontology, 81, 1194-1217; doi: 10.1666/06-056R.1.

Over, D.J. \& Rhodes, M.K. 2000. Conodonts from the Upper Olentangy shale / Upper Devonian, Central Ohio) and the stratigraphy across the Frasnian-Fammenian boundary. Journal of Paleontology, 74, 101-112.

Ovnatanova, N.S. \& Kononova, L.I. 2001. Conodonts and Upper Devonian (Frasnian) biostratigraphy of central regions of Russian Platform. Courier Forschungsinstitut Senckenberg, 233, 1-115.

Ovnatanova, N.S. \& Kononova, L.I. 2008. Frasnian conodonts from the eastern Russian Platform. Journal of Paleontology, 42, 997-1166; doi: 10.1134/S0031030108100018.

Ovnatanova, N.S., Kononova, L.I., Kolesnik, L.S. \& Gatovsky, Y.A. 2017. Upper Devonian conodonts of Northeastern European Russian. Journal of Paleontology, 51, 973-1165; doi: 10.1134/S003103011710001X.

Pander, C.H. 1856. Monographie der fossilen Fische des silurischen Systems der russisch-baltischen Gouvernements. Ph.D. Thesis. Akademie der Wissenschaften. 91 pp.

Perri, M.C. \& Spalletta, C. 1980. Conodonti e biozonatura del Frasniano (Devoniano sup.) di Pramosio, Alpi Carniche. Bollettino della Società Paleontologica Italiana, 19, 281 310.

Purnell, M.A., Donoghue, P.C.J. \& Aldridge, R.J. 2000. Orientation and anatomical notation in conodonts. Journal of Paleontology, 74, 113-122; doi: 10.1017/ S0022336000031292.

Racki, Gr. 1985. Conodont biostratigraphy of the Givetian/ Frasnian boundary beds at Kostomloty in the Holy Cross Mts. Acta Geologica Polonica, 35, 265-275.

Racki, Gr. \& Bultynck, P. 1993. Conodont biostratigraphy of the Middle to Upper Devonian boundary beds in the Kielce area of the Holy Cross Mts. Acta Geologica Polonica, 43, 1-32.

Raven, J.G. 1983. Conodont biostratigraphy and depositional history of the middle Devonian to lower Carboniferous in the Cantabrian Zone (Cantabrian Mountains, Spain). Leidse Geologische Mededelingen, 52, 265-339.

Rejebian, V.A., Harris, A.G. \& Huebner, J.S. 1987. Conodont color and textural alteration: An index to regional metamorphism, contact metamorphism, and hydrothermal alteration. Geological Society of America Bulletin, 99, 471-479.

Rodríguez-Cañero, R. 1993. Contribución al estudio de los Conodontos del Paleozoico del Complejo Maláguide 
Cordillera Bética. Ph.D. Thesis. Universidad de Málaga, España (inédita). 474 pp.

Rodríguez-Cañero, R. \& Guerra-Merchán, A. 1996. Nuevos datos sobre la fauna de conodontos y la edad de la Formación Falcoña (Complejo Maláguide, Cordillera Bética, España). Revista Española de Paleontología, 11, 235-246.

Rodríguez-Cañero, R. \& Martín-Algarra, A. 2014. FrasnianFamennian crisis in the Malaguide Complex (Betic Cordillera, Spain). Terra Nova, 26, 38-54; doi: 10.1111/ ter. 12068 .

Rodríguez-Cañero, R., Martín-Algarra, A., Sarmiento, G.N. \& Navas-Parejo, P. 2010. First Late Ordovician conodont fauna in the Betic Cordillera (South Spain): a palaeobiogeographical contribution. Terra Nova, 22, 330-340.

Roep, T.B. 1972. Stratigraphy of the "Permo-Triassic" Saladilla formation and its tectonics setting in the Betic of Malaga (Vélez Rubio región, SE Spain). Proceedings of the Koninklijke Nederlandse Akademie van Wetenschappen, 75, 223-247.

Roep, T.B. \& Mac Gillavry, H.J. 1960. Preliminary note on the presence of distinct tectonic units in the Betic of Málaga of the Vélez rubio region (SE Spain). Geologie en Mijnbouw, 41, 423-429.

Sánchez de Posada, L.C., Sanz López, J. \& Gozalo, R. 2007. Ostracod and conodont faunal changes across the Frasnian- Famennian (Devonian) boundary at Els Castells, Spanish central Pyrenees. Revue de Micropaleontologie, 51, 205-219; doi: 10.1016/j.revmic.2007.06.001.

Sandberg, C.A., Ziegler, W. \& Bultynck, P. 1989. New standard conodont zones and early Ancyrodella phylogeny across Middle-Upper Devonian boundary. Courier Forschungsinstitut Senckenberg, 110, 195-230.

Sandberg, C.A., Ziegler, W., Dreesen, R. \& Butler, J.L. 1992. Conodont biochronology, biofacies, taxonomy, and event stratigraphy around Middle Frasnian Lion Mudmound (F2h), Frasnes, Belgium. Courier Forschungsinstitut Senckenberg, 150, 1-87.

Sanz de Galdeano, C., Delgado, F. \& López-Garrido, A.C. 1995. Unidades Alpujárrides y Maláguides al NE de Granada (Cordillera Bética). Geogaceta, 18, 27-29.

Sanz-López, J. 2002. Devonian and Carboniferous preStephanian rocks from Pyrenees. In: Palaeozoic Conodonts from Northern Spain (eds. García-López, S. \& Bastida, F.). Cuadernos del Museo Geominero, 1, 367-389.

Sanz-López, J., García-López, S. \& Montesinos, J.R.T. 1999. Conodontos del Frasniense superior y Fameniense inferior de la Formación Cardaño (Unidad de Gildar-Montó, Dominio Palentino, Zona Cantábrica). Revista Española de Paleontología, 14, 25-35.

Soboleva, M.A. \& Sobolev, D.B. 2015. Biostratigraphic characteristics based on conodonts of the Middle-Upper
Frasnian beds of the Malaya Usa River (Polar Urals). Vestnik IG Komi Nauchnogo Tsentra Ural Rossiiskoi Akademii Nauk, 6, 15-28.

Stauffer, C.R. 1938. Conodonts of the Olentangy Shale. Journal of Paleontology, 12, 411-443.

Stauffer, C.R. 1940. Conodonts from the Devonian and associated clays of Minnesota. Journal of Paleontology, $14,417-435$.

Sweet, W.C. 1988. The Conodonta. Oxford monographs on Geology and Geophysics, Clarendon Press.

Sweet, W.C. \& Donoghue, P.C.J. 2001. Conodonts: Past, present, future. Journal of Paleontology, 75, 1174-1184; doi: 10.1017/S0022336000017224.

Talent, J.A., Gaetani, M., Mawson, R., Molloy, P.D. \& Conaghan, P.J. 1999. Early Ordovician and Devonian conodonts from the western Karakoran and Hindu Kush, Northernmost Pakistan. Rivista Italiana di Paleontologia e Stratigrafia, 105, 1-8; doi: 10.13130/2039-4942/5373.

Ulrich, E.O. \& Bassler, R.S. 1926. A classification of the toothlike fossils, conodonts, with description of American Devonian and Mississippian species. Proceedings of the United States National Museum, 68, 1-63.

Uyeno, T.T. 1967. Conodont zonation, Waterways Formation (Upper Devonian), northeastern and central Alberta. Geological Survey of Canada, Paper, 67-30, 1-21; doi: 10.4095/100913.

Uyeno, T.T. 1990. Biostratigraphy and conodont fauna of Upper Ordovician through Middle Devonian rocks, Eastern Artic Archipelago. Geological Survey of Canada Bulletin, 401, 1-211.

Van de Boogaard, M. 1965. Two conodont fauna from the Paleozoic of the Betic of Malaga near Velez Rubio S.E. Spain. Koninklijke Nederlandse Akademie van Wetenschappen, Series B, 68, 33 - 37.

Van de Boogaard, M. 1983. Conodont faunas from Portugal and southwestern Spain. Part. 7. A Frasnian conodont fauna near the Estação de Cabrela (Portugal). Scripta geologica, 69, 1-17.

Weddige, K. 1977. Die Conodonten der Eifel-Stufe im Typusgebiet und in benachbarten Faziesgabieten. Senckenbergiana lethaea, 58, 271-419.

Ziegler, W. 1973. Catalogue of Conodonts. E. Schweizerbart'sche Verlagsbuchhandlung, I, 504.

Ziegler, W. 1977. (Editor). Catalogue of Conodonts. E Schweizerbart'sche Verlagsbuchhandlung, III, 574.

Ziegler, W. \& Sandberg, C.A. 1990. The Late Devonian standard conodont zonation. Courier Forschungsinstitut Senckenberg, 121, 1-115.

Ziegler W., Klapper G. \& Johnson J. G. 1976. Redefinition and subdivision of the varcus-Zone (Conodonts, Middle-?) Upper Devonian in Europe and North America. Geology and Palaeontology, 10, 109-140. 